\title{
Identification of inflammatory response and alternative splicing in acute kidney injury and experimental verification of the involvement of RNA-binding protein RBFOX1 in this disease
}

\author{
FANGYOU LIN* ${ }^{*}$ LEI XU* , RUN YUAN* ${ }^{*}$, SHANGTING HAN, JINNA XIE, KUN JIANG, \\ BOJUN LI, WEIMIN YU, TING RAO, XIANGJUN ZHOU and FAN CHENG \\ Department of Urology, Renmin Hospital of Wuhan University, Wuhan, Hubei 430060, P.R. China
}

Received November 15, 2021; Accepted December 22, 2021

DOI: $10.3892 /$ ijmm.2022.5087

\begin{abstract}
An increasing number of inflammatory responses and alternative splicing (AS) have been recently reported to be associated with various kidney diseases. The effect of inflammatory response on acute kidney injury (AKI) has not been fully clarified. In the present study, a mouse model of AKI induced by cisplatin and ischemia-reperfusion (IR) was established and genome-wide profiling analysis and identification of differentially expressed genes (DEGs) in kidney tissue was conducted by Gene Ontology (GO) functional analysis, Kyoto Encyclopedia of Genes and Genomes (KEGG) pathway enrichment analysis, protein-protein interaction (PPI) network analysis and RT-qPCR. The results revealed that common DEGs in AKI induced by cisplatin and IR were enriched in the inflammatory response pathway, including hub genes CSF-1, CXCL1, CXCL10, IL-1 $\beta$, IL-34, IL-6 and TLR2. AS in AKI was initially reported. Cisplatin-induced AS was enriched in the phosphorylation pathway, involving regulated AS genes CSNK1A1, PAK2, CRK, ADK and IKBKB. IR-induced AS was enriched in apoptosis and proliferation pathways, including DEGs ZDHHC16, BCL2L1 and FGF1 regulated by AS. The ability of RNA-binding proteins (RBPs) to regulate AS was coordinated with the function of context-dependent genetic mechanisms. A total of 49 common differentially expressed RBP genes were screened. RNA binding fox-1 homolog 1 (RBFOX1) was revealed to be the top downregulated gene. The relative levels of RBFOX1 in the nuclei of mouse renal tubular epithelial cells in mRNA and proteins were downregulated
\end{abstract}

Correspondence to: Dr Xiangjun Zhou or Dr Fan Cheng, Department of Urology, Renmin Hospital of Wuhan University, 99 Zhang Road, Wuchang, Wuhan, Hubei 430060, P.R. China

E-mail: zxj19840902@163.com

E-mail: urology1969@aliyun.com

*Contributed equally

Key words: acute kidney injury, inflammation, alternative splicing, RNA-binding protein, RNA binding fox-1 homolog 1 by cisplatin and IR. Moreover, the biological functions of RBFOX1 were investigated in human renal proximal tubular epithelial cells (HK-2 cells). Results of in vitro experiments revealed that exogenous RBFOX1 inhibited inflammation and oxidative stress to reduce hypoxia/reoxygenation-induced apoptosis of HK-2 cells. This phenomenon may be related to the inhibition of NF- $\kappa \mathrm{B}$ and the activation of the NRF2/HO-1 signaling pathway. In conclusion, the inflammatory cytokines, AS and RBPs in AKI were analyzed in the present study via whole transcriptome sequencing. It was revealed that the RBP gene RBFOX1 was involved in the pathogenesis of AKI. Thus, the present study provided novel insights into the mechanism of AKI pathogenesis.

\section{Introduction}

Acute kidney injury (AKI) is a frequent clinical emergency associated with various aetiologies and pathophysiological processes leading to decreased glomerular filtration function, and is considered an independent predictor of mortality in hospitalized patients, especially for high-risk patients. Approximately 1.7 million individuals succumb to AKI worldwide every year $(1,2)$. The most common causes of AKI include ischemia, hypoxia, or nephrotoxicity (3). An underlying feature is a rapid decline in glomerular filtration rate usually associated with decreases in renal blood flow. The precise cause underlying the pathology of the disease remains unknown. Identification of gene signatures associated with disease onset and progression would help to improve understanding of the molecular mechanisms involved in the disease pathogenesis.

Pathogenically, AKI is generally described as the injury of renal tubular epithelial cells and vasculature, accompanied by the activation of a robust inflammatory response (4). Inflammation response has been recognized to play an important role in ischemic and toxic models of AKI $(5,6)$. In experimental models, tubular cell injury and death have been revealed to be directly prevented by suppressing inflammation (7).

Alternative splicing (AS) allows mRNA to produce different protein subtypes, which represents an important post-transcriptional regulatory mechanism for gene expression 
and greatly expands gene coding capabilities, protein diversity and biology function in eukaryotic organisms (8). AS is a highly-regulated process performed by an intricate molecular machine-spliceosome; any fault in this regulation can result in cellular dysfunction and disease (9). More than 2,000 splicing mutation disease entities are known, resulting in 370 diseases and involving 303 genes (10). An increasing number of splice isoforms have been previously reported to be associated with various kidney diseases (11). Alternative splicing events (ASEs) are largely controlled by RNA-binding proteins (RBPs) that recognize specific regulatory sequences embedded in the pre-mRNA transcripts and coordinate with the function of context-dependent genetic mechanisms (12).

Inflammation, AS and RBPs in cisplatin and ischemia-reperfusion (IR)-induced AKI was identified. In vitro experiments verified that the RBP gene RNA binding fox-1 homolog 1 (RBFOX1) was involved in the pathogenesis of AKI. This comprehensive analysis may reveal the expression of inflammation, AS and RBPs in AKI and the role of RBFOX1 in this disease. In conclusion, the present study provided novel insights into the pathogenesis of AKI.

\section{Materials and methods}

Establishment of the AKI mouse model. A total of 30 male C57BL/6J mice (six to eight weeks old; 20-25 g) were purchased from Beijing Vital River Laboratory Animal Technology Co., Ltd. All mice were adaptively reared for one week in a standardized environment with a 12-h light/dark cycle and a controlled $20-26^{\circ} \mathrm{C}$ temperature and $40-70 \%$ humidity. Access to food and water was ad libitum. Mice were randomly divided into three groups ( $\mathrm{n}=10 /$ group): Control, cisplatin and IR. After the C57BL/6J mice were adapted to the environment for one week, the AKI model was established by a single injection of cisplatin and IR. In accordance with a previous study on the dosage and duration of cisplatin (13), a cisplatin-induced AKI model was established by intraperitoneal injection (at approximately $0.5 \mathrm{~cm}$ on both sides of the midline of the hypogastrium) of cisplatin (cat. no. HY-17394; MedChemExpress) dissolved in physiological saline at a single dose of $30 \mathrm{mg} / \mathrm{kg}$ body weight for $24 \mathrm{~h}$. Under a 3-4\% induction dose and 1-2\% maintenance dose of isoflurane anesthesia, the bilateral renal pedicles were clamped through a midline abdominal incision for $30 \mathrm{~min}$ and blood flow was restored for $24 \mathrm{~h}$ to establish an AKI model induced by severe IR injury (14). The control mice were not given special intervention under the same feeding conditions. The vital signs of the treated mice were observed every $6 \mathrm{~h}$. Within $24 \mathrm{~h}, 3$ mice in the cisplatin group and 1 mouse in the IR group succumbed. Death was verified by applying pressure on the mouse nail bed (toe-pinch reflex). After $24 \mathrm{~h}$, all mice were sacrificed under isoflurane anesthesia, $0.5-0.6 \mathrm{ml}$ blood was collected from the heart and both kidneys were dissected after cardiac perfusion with physiological saline solution. All animal procedures in the present study were approved (approval no. WDRM-20200904) by the Ethics Review Committee of Animal Welfare of Renmin Hospital of Wuhan University (Wuhan, China) and in accordance with the National Institutes of Health Guide for the Care and Use of Laboratory Animals.
Renal function assessment. The supernatant was collected after the blood samples were centrifuged at $4^{\circ} \mathrm{C}$ and $1,000 \mathrm{x} \mathrm{g}$ for $15 \mathrm{~min}$. According to the protocol of the kits provided by the manufacturer (cat. nos. K066 and K072a; Changchun Huili Biotech Co., Ltd.), serum creatinine ( $\mathrm{Cr}$ ) and blood urea nitrogen (BUN) levels, respectively, were detected using an automatic biochemical analyzer (Rayto Life and Analytical Sciences Co., Ltd.).

Histopathological studies and immunohistochemical staining. The kidney tissue was fixed in $4 \%$ paraformaldehyde for $24 \mathrm{~h}$ at room temperature and paraffin embedding and then cut into $4-\mu \mathrm{m}$-thick sections and placed on a glass slide. For periodic acid-Schiff (PAS) staining, the slices were placed in PAS dye solution staining for $45 \mathrm{~min}$ at $37^{\circ} \mathrm{C}$. In accordance with the terminal deoxynucleotidyl transferase dUTP nick-end labeling (TUNEL) kit protocol provided by the manufacturer (cat. no. G1507; Wuhan Servicebio Technology Co., Ltd.), the tissue sections were incubated in the reaction solution at $37^{\circ} \mathrm{C}$ for $1 \mathrm{~h}$. At $37^{\circ} \mathrm{C}$, the nucleus was stained in 3,3N-diaminobenzidine tertrahydrochloride and developed for 30 min followed by counterstaining in hematoxylin staining solution for $1 \mathrm{~min}$. The sections were then briefly dried and mounted with resin mounting medium. The tissue and cell sections were blocked with $5 \%$ goat serum at room temperature for $30 \mathrm{~min}$ and then incubated with rabbit anti-RBFOX1 (1:1,000; cat. no. ab254413; Abcam) overnight at $4^{\circ} \mathrm{C}$ and goat anti-rabbit secondary antibody (1:100; cat. no. GB21303; Wuhan Servicebio Technology Co., Ltd.) labeled with sulfo-Cyanine3 dye at room temperature for $30 \mathrm{~min}$; finally, the nuclei were counterstained using $2 \mu \mathrm{g} / \mathrm{ml}$ 4',6-diamidino-2-phenylindole at room temperature for $10 \mathrm{~min}$. A total of 10 fields of view were randomly selected from each slice under an optical fluorescence microscope for evaluation and analysis (Olympus Corporation; magnification, x200). The percentage of damaged renal tubules was calculated in accordance with the scoring criteria as follows: 0 , no damage; $1,<25 \% ; 2,25-49 \% ; 3,50-75 \% ; 4,>75 \%$ (15). Semiquantitative analysis of the positive staining area of apoptotic cells was conducted using ImageJ software (v1.8.0; National Institutes of Health).

RNA extraction and sequencing. The kidney tissues were ground into fine powder prior to RNA extraction. Total RNA was extracted with TRIzol ${ }^{\circledR}$ (cat. no. 15596-026; Invitrogen; Thermo Fisher Scientific, Inc.). The RNA was further purified with two phenol-chloroform treatments and then treated with RQ1 DNase (cat. no. M6101; Promega Corporation) to remove DNA. The quality and quantity of the purified RNA were redetermined by measuring the absorbance at $260 \mathrm{~nm} / 280 \mathrm{~nm}$ (A260/A280) using Smartspec Plus (Bio-Rad Laboratories, Inc.). The integrity of RNA was further verified by 1.5\%-agarose gel electrophoresis.

For each sample, $1 \mu \mathrm{g}$ of the total RNA was used for RNA sequencing (RNA-seq) library preparation by VAHTS Stranded mRNA-seq Library Prep kit (cat. no. NR602; Vazyme Biotech, Co., Ltd.). Polyadenylated mRNAs were purified and fragmented and then converted into double strand cDNA. After the step of end repair and a-tailing, the DNAs were ligated to VAHTS RNA adapters (cat. no. NR803; Vazyme Biotech Co., 
Ltd.). Purified ligation products corresponding to 200-500 bps were digested with heat-labile uracil-DNA glycosylase (UDG) and the single-strand cDNA was amplified, purified, quantified and stored at $-80^{\circ} \mathrm{C}$ prior to sequencing.

For high-throughput sequencing, the libraries were prepared following the manufacturer's protocol and were applied to Illumina HiSeq X Ten system for 150 nucleotides (nt) paired-end sequencing by HiSeq X Ten Reagent kit v2.5 (cat. no. FC-501-2501; Illumina, Inc.) (GEO accession code: GSE142138 (16); URL: https:/www.ncbi.nlm.nih.gov/geo/ query/acc.cgi?acc $=$ GSE142138).

RNA-Seq raw data clean and alignment. Raw reads containing more than 2-N bases were initially discarded. Then, adaptors and low-quality bases were trimmed from raw sequencing reads using FASTX-Toolkit (v0.0.13) (http://hannonlab.cshl. edu/fastx_toolkit/). The short reads less than 16 nt were also dropped. Subsequently, clean reads were aligned to the GRch38 genome by TopHat2, allowing four mismatches (17). Uniquely mapped reads were used for gene read number counting and fragments per kilobase of exon model per million mapped fragment (FPKM) calculation (18).

Differentially expressed gene analysis. The R Bioconductor package edgeR was utilized to screen out the differentially expressed genes (DEGs) and common DEGs (co-DEGs) (19). A false discovery rate (FDR) $<0.05$ and fold change $>2$ or $<0.5$ were set as the cut-off criteria for identifying DEGs. The intersections of upregulated and downregulated DEGs in AKI tissues induced by cisplatin and IR were obtained for further functional enrichment analysis.

AS analysis. The ASEs and regulated alternative splicing events (RASEs) between the samples were defined and quantified using the ABLas pipeline as previously described (20). In summary, ABLas detection of 10 types of ASEs was based on the splice junction reads, including exon skipping (ES), alternative 5' splice site (A5SS), alternative 3' splice site (A3SS), intron retention, mutually exclusive exons, mutually exclusive 5'UTRs, mutually exclusive 3'UTRs, cassette exon, A3SS\&ES and A5SS\&ES.

To assess the dysregulation of ASEs, an unpaired Student's t-test was performed to evaluate the significance of the ratio alteration of $\mathrm{AS}$ events. Events, which were significant at $\mathrm{P}$-value cutoff corresponding to a false discovery rate cutoff of 5\%, were considered dysregulated ASEs. The overlapping regulated alternative splicing genes (RASGs) induced by cisplatin and IR were obtained using Venn diagram tool.

Reverse transcription-quantitative polymerase chain reaction (RT-qPCR) validation of DEGs and ASEs. RT-qPCR was performed for certain DEGs. The primer sequences are presented in Table I. Total RNA remaining from RNA-seq library preparation was used for RT-qPCR. RNA was reverse transcribed into cDNA using an M-MLV reverse transcriptase assay kit according to the manufacturer's protocol (cat. no. R021-01; Vazyme Biotech Co., Ltd.). qPCR was performed with the StepOne real-time PCR system using the SYBR Green PCR Reagent kit (cat. no. 11143ES50; Shanghai Yeasen Biotechnology Co., Ltd.). The thermocycling
Table I. Primers used for reverse transcription-quantitative PCR.

Gene $\quad$ Primer sequence (5'-3')

$\begin{array}{ll}\text { KIM-1 } & \text { F: CCAGGCGCTGTGGATTCTTA } \\ & \text { R: TGTACCGACTGCTCTTCTGATAGG } \\ \text { NGAL } & \text { F: CAGAGCTACAATGTGCAAGTGGC } \\ & \text { R: CAGCTCCTTGGTTCTTCCATACA } \\ \text { RBFOX1 } & \text { F: TCATTTAGAGCAAGTGGGTG } \\ & \text { R: TCTGGGTTTGCAGATTGAGT } \\ \text { CSF-1 } & \text { F: GCACACAGGGGCGGCT } \\ & \text { R: GCATTCTCTACCCCTCCACCT } \\ \text { IL-1 } 3 & \text { F: ATGTGCTGGTGCTTCATTCA } \\ & \text { R: AGCCCGCACTGAGGTCTTTC } \\ \text { IL-6 } & \text { F: CCCCAATTCCAATGCTCTCC } \\ & \text { R: CGCACTAGGTTTGCCGAGTA } \\ \text { TLR2 } & \text { F: AGTCAGGAACTGGGTGGAGA } \\ & \text { R: ACCAAGACCTACCTGGAGTG } \\ \text { CXCL1 } & \text { F: CTGTGCTAGTAGAAGGGTGTTGT } \\ & \text { R: ACGAGACCAGGAGAAACAGG } \\ \text { IL-34 } & \text { F: GATACGGCATTTGGTTGGTC } \\ & \text { R: GGCTGGTTGCTATCCCTTACA } \\ \text { CXCL10 } & \text { F: ACCCATTGATACATACTTGA } \\ & \text { R: GTAAGGACGAACTTAACCAC } \\ \text { LGALS3 } & \text { F: TAACCACGCCATGATCTAAG } \\ & \text { R: GCAAAGTTTCCCACTCCTAA } \\ \text { IKBKB } & \text { M-F: TTCACCTGTCGCCTGATGGC }\end{array}$

IKBKB M-F: TTCACCTGTCGCCTGATTGTGC AS-F: CCTCTCAAGAACCTGATTGTGC M/AS-R: GCCTGGGAAATGAAAGAACG

CRK M/AS-F: TCAAGGCAGGGTAGTGGAGT AS-R: AACCATCAGGAGCTCCAATCAG M-R: CAGCTCACCGACCTCCAATCAG

ADK M/AS-F: GGCAGAGCCAAACTACGGTG AS-R: GACCCAGATAATCTCAGCGCTT M-R: AGCACATTTTCACTCAGCGCTT

PAK2 M-F: CTTTTTACTTCCTTTTTCTGTGC AS-F: CAGTCCAGGCCTTTTTCTGTGC M/AS-R: TCCAGTTCGGATGAGCAGTA

CSNK1A1 M/AS-F: AGCCTCTTCCAGTGGGCAGGGTCA AS-R: TCTTTAATACCTGTGGGGGT M-R: GCTTAGAAACCTGTGGGGGT

BCL2L1 M/AS-F: GCTCACTTACTGGGTCTGCT AS-R: AGCAATTCTGAACCTTATCT M-R: GCAGTCAGCCAGAACCTTATCT

SHF M/AS-F: TTTTGCTGGTCTCACTGTTG AS-R: CTGGAAAACCAGCTGGTACCAC M-R: GGAAAACCAGGTCTGGTACCAC

FGF1 M-F: CTGTCCCTTGTCCCATCCAC AS-R: ATATCTGACCTGTGCTGAGCCT M/AS-R: CCAGTTCTTCAGTGCTGAGCCT

ZDHHC16 M/AS-F: TAACCATCGCTACTTCTTCTC AS-R: GTTTCATTTTCTCAATGGCA M-R: CTGGTGGTACGTCTCAATGGCA

GAPDH F: GGAGATGCTCAGTGTTGG R: TGACAATGAATACGGCTACA

F, forward; R, reverse; M, model; AS, alternative splicing. 
conditions were as follows: Initial denaturation at $95^{\circ} \mathrm{C}$ for $10 \mathrm{~min}, 40$ cycles of denaturation at $95^{\circ} \mathrm{C}$ for $15 \mathrm{sec}$ and annealing and extension at $60^{\circ} \mathrm{C}$ for $1 \mathrm{~min}$. PCR amplifications were performed in triplicate for each sample. The RNA expression levels of all the genes were normalized against those of GAPDH using the $2^{-\Delta \Delta C q}$ method (21).

RT-qPCR assay was also performed for ASE validation. A boundary-spanning primer was used for the sequence encompassing the junction of the constitutive exon and alternative exon as well as an opposing primer in a constitutive exon to detect alternative isoforms. The boundary-spanning primer of alternative exon was designed according to 'model exon' to detect model splicing or 'altered exon' to detect altered splicing (22).

Functional enrichment analysis. Gene Ontology (GO) terms (23) and Kyoto Encyclopedia of Genes and Genomes (KEGG) pathways (24) were identified using KOBAS 2.0 server (25) to sort out functional categories of DEGs. Hypergeometric test and Benjamini-Hochberg false discovery rate (FDR)-controlling procedure were used to define the enrichment of each term.

Protein-protein interaction (PPI) network construction and module analysis. The Search Tool for the Retrieval of Interacting Genes (http://www.string-db.org) was used to analyze the association between DEGs that are co-, up- or downregulated by cisplatin and IR. The PPI network was visualized and constructed using Cytoscape v3.8.2 (26).

Cell culture and hypoxia/reoxygenation (H/R) model preparation. Human renal proximal tubular epithelial cells (HK-2 cells) were obtained from Procell Life Science and Technology Co., Ltd. The identification of the HK-2 cell lines was conducted at the China Centre for Type Culture Collection. The HK-2 cells were cultured in DMEM/F12 medium (Gibco; Thermo Fisher Scientific, Inc.) containing 10\% fetal bovine serum in a humidified atmosphere of $95 \% \mathrm{O}_{2}$ and $5 \% \mathrm{CO}_{2}$ at $37^{\circ} \mathrm{C}$. The HK-2 cells were placed on pre-hypoxia-treated glucose-free and serum-free medium and then cultured in a three-gas incubator $\left(1 \% \mathrm{O}_{2}, 4 \% \mathrm{CO}_{2}\right.$ and $\left.95 \% \mathrm{~N}_{2}\right)$ at $37^{\circ} \mathrm{C}$ for $24 \mathrm{~h}$ (27). Finally, the cells were cultured in a normal medium and then reoxygenated in a normal incubator for 1, 3 and $6 \mathrm{~h}(28)$.

Cell transfection experiment. The RBFOX1 plasmid [pEnC MV-RBFOX1(human)-3xFLAG-SV40-Neo] and the corresponding vector control (pEnCMV-MCS-3xFLAG-SV40-Neo) were obtained from Wuhan MiaoLing Biotechnology, Co., Ltd. (www.miaolingbio.com). HK-2 cells $\left(1 \times 10^{6}\right)$ were seeded in 6-well culture plates. After the cells reached $60-70 \%$ confluence, RBFOX1 plasmid or vector control $(5 \mu \mathrm{g})$ was mixed with $5 \mu$ l Lipofectamine ${ }^{\circledR} 2000$ transfection reagent (Invitrogen; Thermo Fisher Scientific, Inc.) in serum-free medium to prepare the transfection complex. The mixing system was incubated at room temperature for $15 \mathrm{~min}$ and then uniformly added to each group of cells at $37^{\circ} \mathrm{C}$ for $24 \mathrm{~h}$. The transfected cells were treated with normal fresh medium for 4-6 $\mathrm{h}$ and then subjected to further experiments. The transfection efficiency was determined using western blotting.
Flow cytometric analysis. Following digestion with trypsin, the cells reached $70-80 \%$ confluence in 6-well culture plates were centrifuged at $300 \mathrm{x} \mathrm{g}$ for $5 \mathrm{~min}$ at room temperature. After washing with PBS, $100 \mu 1$ of $1 \mathrm{X}$ binding buffer was added to resuspend the cells. Then, $5 \mu \mathrm{l}$ Annexin V-FITC and $10 \mu \mathrm{l}$ PI (BD Biosciences) staining solution were added. The cells were incubated at room temperature for $20 \mathrm{~min}$ in the dark. Finally, $400 \mu \mathrm{l}$ of $1 \mathrm{X}$ binding buffer was added to resuspend the cells in the solution. Flow cytometric detection was completed within $1 \mathrm{~h}$ using CytoFLEX flow cytometer (Beckman Coulter, Inc.). FlowJo software (version 10.8.0; BD Biosciences) was used for flow cytometric data analysis.

ELISA. The levels of inflammatory factors in the cell supernatant were measured by TNF- $\alpha$, IL- 6 and IL- $1 \beta$ ELISA kits (cat. nos. BMS223-4, BMS213-2 and BMS224-2, respectively; eBiosience; Thermo Fisher Scientific, Inc.) in accordance with the manufacturer's protocol.

Measurement of reactive oxygen species (ROS) production. The cells were collected and treated with diluted $10 \mu \mathrm{mol} / \mathrm{l}$ DCFH-DA probe (Beyotime Institute of Biotechnology) and then incubated at $37^{\circ} \mathrm{C}$ for $20 \mathrm{~min}$. The difference in ROS content in each group was determined by flow cytometry.

Measurement of the levels of superoxide dismutase (SOD) and malondialdehyde (MDA) activity. Activities of SOD and MDA were detected following the protocols of the manufacturers of the corresponding kits (cat. nos. A001-3-2 and A003-1-2; Nanjing Jiancheng Bioengineering Institute). Absorbance values at wavelengths of 550 and $532 \mathrm{~nm}$ were measured using a microplate reader to detect the activities of SOD and MDA in the cells.

Western blot analysis. Total protein was extracted from the kidney tissue or HK-2 cells using RIPA lysis buffer (BioSharp LifeSciences, Inc.) and the protein concentration was determined using BCA Protein assay reagent (Thermo Fisher Scientific, Inc.). The same amount of protein (15-30 $\mu \mathrm{g})$ in each well was subjected to $10-12 \%$ SDS-PAGE. The transfer polyvinylidene difluoride (PVDF) (cat. no. IPVH00010; Millipore, Inc.) membrane was blocked with $5 \%$ non-fat milk in Tris-buffered saline containing $0.1 \%$ Tween-20 at room temperature for $1 \mathrm{~h}$ and then incubated with primary antibodies at $4^{\circ} \mathrm{C}$ overnight: anti-RBFOX1 (1:1,000; cat. no. ab254413), anti- $\beta$-actin (1:1,000; cat. no. ab8226; both from Abcam), anti-NF-кB (p65; 1:1,000; cat. no. AF5006), anti-phospho-NF-кB (p-p65; 1:1,000; cat. no. AF2006; both from Affinity Biosciences), anti-NRF2 (1:1,000; cat. no. 16396-1-AP) and anti-HO-1 (1:1,000; cat. no. 10701-1-AP; all from ProteinTech Group, Inc.). After washing thrice with Tris Buffered Saline with Tween for 5 min each time, the membranes were incubated with the horseradish peroxidase-conjugated goat anti-mouse IgG $(1: 1,000$; cat. no. ab205719) and goat anti-rabbit $\operatorname{IgG}(1: 1,000$; cat. no. ab205718; both from Abcam) secondary antibodies at room temperature for $1 \mathrm{~h}$. Target bands were visualized using enhanced chemiluminescent (ECL) kit (cat. no. BL523B; Biosharp Life Sciences, Inc.). The ChemiDoc Imaging System (Bio-Rad Laboratories, Inc.) was used to detect chemiluminescence blots. ImageJ software (version 1.8.0; National Institutes 

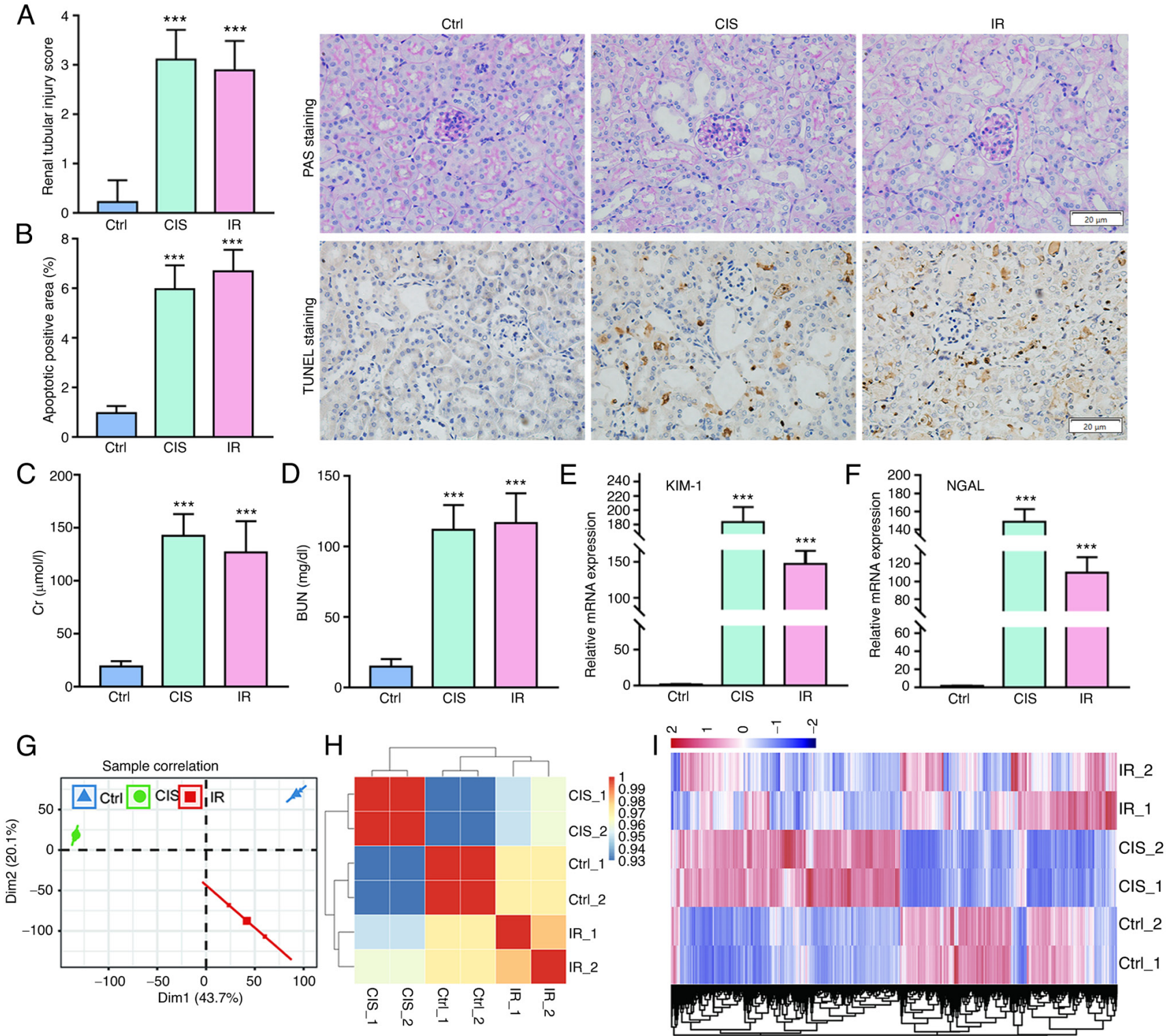

Figure 1. Construction of mouse acute kidney injury model and genome-wide analysis of kidney tissue. (A and B) PAS and TUNEL staining of kidney tissues. (C) Level of serum Cr. (D) Level of BUN. (E and F) mRNA expression of (E) KIM-1 and (F) NGAL in kidney tissue. (G) Principal component analysis and $(\mathrm{H})$ sample correlation analysis of kidney tissue samples in groups CIS, IR and control. (I) Heatmap of DEGs among CIS, IR and control groups ${ }^{* * * *} \mathrm{P}<0.05$ vs. Ctrl. CIS, cisplatin; IR, ischemia-reperfusion; DEGs, differentially expressed genes; Cr, creatinine; BUN, blood urea nitrogen.

of Health) was used to semi-quantify protein expression with $\beta$-actin as the loading control.

Statistical analysis. Data are presented as the mean \pm SD from three or more independent experiments. GraphPad Prism 7 (GraphPad Software, Inc.) was used for statistical analysis. Continuous variables were analyzed using one-way ANOVA and unpaired Student's t-test. Enumerated variables were analyzed using chi-square and Fisher's exact tests. Bonferroni's test was used for post hoc comparisons. All experiments were independently repeated in triplicate. A two-sided $\mathrm{P}<0.05$ was considered to indicate a statistically significant difference.

\section{Results}

Construction and genome-wide profiling of AKI model induced by cisplatin and IR. Mouse AKI models induced by cisplatin and IR were successfully constructed. It was revealed that cisplatin and IR caused the kidney tissue to become severely damaged and increase in cell apoptosis (Fig. 1A and B). The levels of $\mathrm{Cr}$ and BUN and the mRNA expression of KIM-1 and NGAL in kidney tissue were significantly increased, indicating that cisplatin and IR caused serious damage to the kidney function of mice compared with those in the controls (Fig. 1C-F).

The principal component analysis and sample correlation analysis revealed that samples treated with cisplatin and IR can be separated from control samples (Fig. 1G and H). The hierarchical clustering heat map revealed that the gene expression profiles of the cisplatin group and the IR group were evidently different from those of the control group (Fig. 1I).

Co-DEGs in AKI induced by cisplatin and IR are involved in the inflammatory response pathway. A total of 980 
A
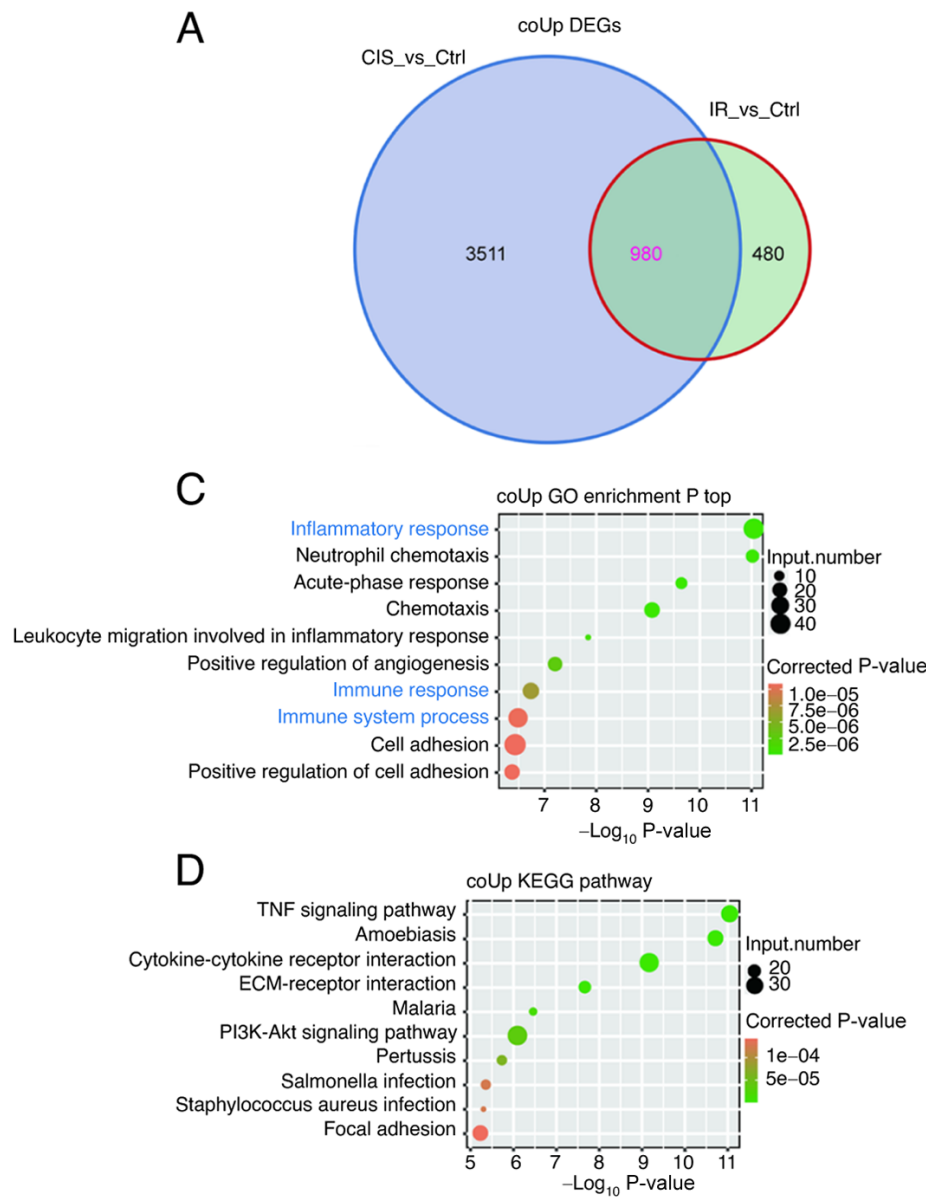

B
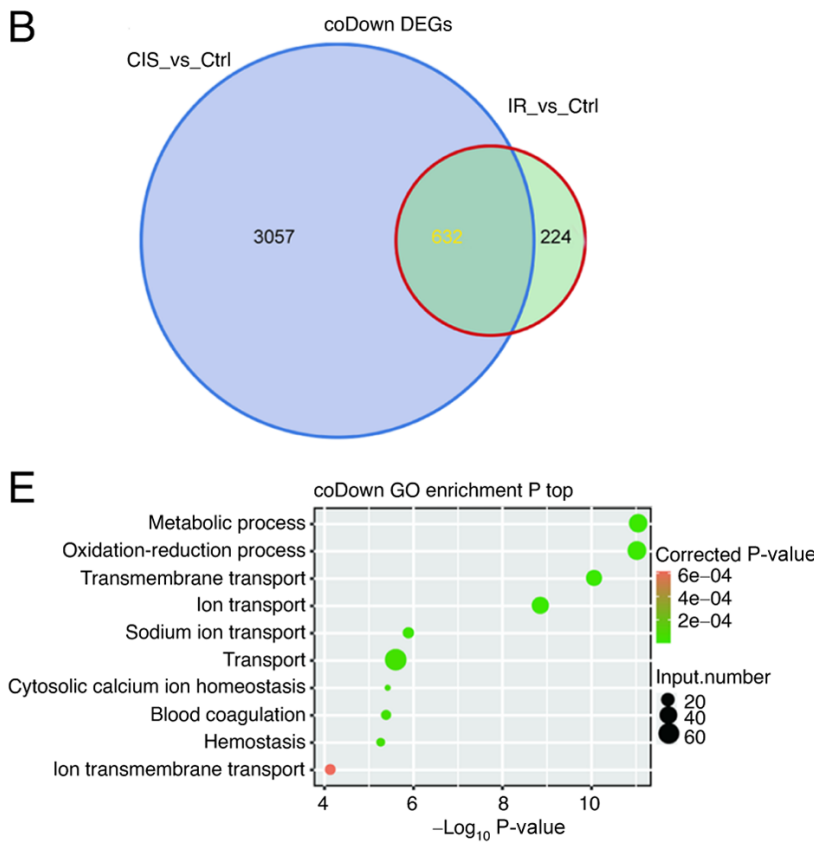

$\mathrm{F}$

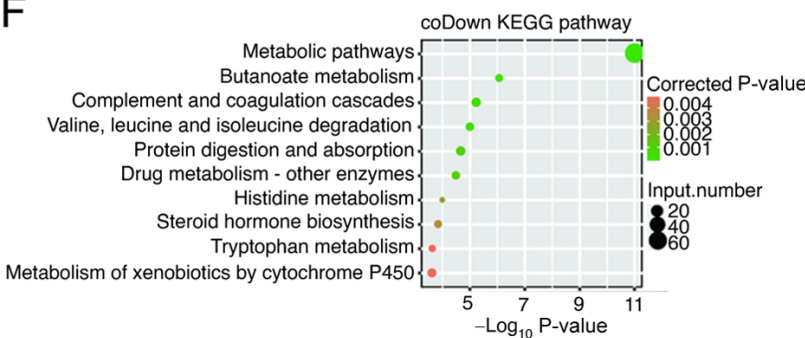

Figure 2. GO and KEGG analyses of co-DEGs in acute kidney injury induced by CIS and IR. (A and B) Venn diagram revealing the number of co-upregulated and co-downregulated DEGs. (C) GO analysis and (D) KEGG analysis revealed the most enriched top 10 terms and pathways of the co-upregulated DEGs. (E) GO analysis and (F) KEGG analysis revealed the most enriched top 10 terms and pathways of the co-downregulated DEGs. GO, Gene Ontology; co-DEGs, common differentially expressed genes; CIS, cisplatin; IR, ischemia-reperfusion.

co-upregulated DEGs and 632 co-downregulated DEGs were identified in AKI induced by cisplatin and IR (Fig. 2A and B). The co-upregulated DEGs were mainly enriched in acute immune and inflammatory response (Fig. 2C and D). The co-downregulated DEGs were mainly related to cell metabolism and functional damage (Fig. 2E and F).

The PPI network of co-upregulated DEGs revealed that IL-6, TNF, MMP9, TLR2, IL-1 $\beta$, TIMP1, CCL2, CXCL1, CXCL10, CSF-1 and LGALS3 were among the top 40 hub genes (Fig. S1). In addition, further RT-qPCR results revealed that the inflammatory genes CSF-1, CXCL1, CXCL10, IL-1 $\beta$, IL-34, IL-6 and TLR2 were upregulated in AKI induced by cisplatin and IR (Fig. 3). These findings suggested that the inflammation response pathway plays an important role in the pathogenesis of AKI. In addition, the PPI network of co-downregulated DEGs suggested multiple key genes including RBFOX1 (Fig. S2).

Transcriptome analysis of AS in AKI induced by cisplatin and $I R$. The heat map of the transcriptome analysis revealed that the differentially expressed profile of RASGs induced by cisplatin was evidently different from that of IR (Fig. 4A). The types of RASEs were mainly concentrated in A3SS, A5SS, ES and cassette exon events (Fig. 4B). The number of differences in RASGs between the cisplatin and IR groups was markedly higher than the number of intersections (Fig. 4C). Cisplatin-induced RASGs were highly enriched in phosphorylation and related cell signaling pathways (Fig. 4D-F) and IR-induced RASGs were related to cell metabolism, apoptosis and phosphorylation (Fig. 4G and H). These findings indicated that DEGs regulated by AS may play an important role in AKI through the phosphorylation and apoptosis pathways.

Examples and verification of ASEs in AKI induced by cisplatin and IR. The specific structures and processes of the A3SS, cassette exon and A5SS events were demonstrated and the expression of nine mRNA splicing isoforms in cisplatin and IR-induced AKI was verified by RNA-seq quantification and RT-qPCR (Figs. 5 and 6). Specifically, cisplatin induced upregulation of CSNK1A1, ADK, CRK, PAK2 and IKBKB genes, which underwent A3SS events and IR caused upregulation of ZDHHC16 (cassette exon), BCL2L1 (A5SS) and FGF1 (A5SS) genes. RNA-seq showed that IR induced the upregulation of SHF (A5SS) gene, but this finding was not confirmed in subsequent RT-qPCR.

Expression profile of RBPs and in vivo validation of RBFOXI in cisplatin and IR-induced AKI. As demonstrated in the heat map, 49 RBP-related genes were found in co-DEGs induced by cisplatin and IR (Fig. 7A). Among the upregulated genes, the 
$\square$ Ctrl $\square$ CIS $\square$ IR Inflammatory response

CSF-1
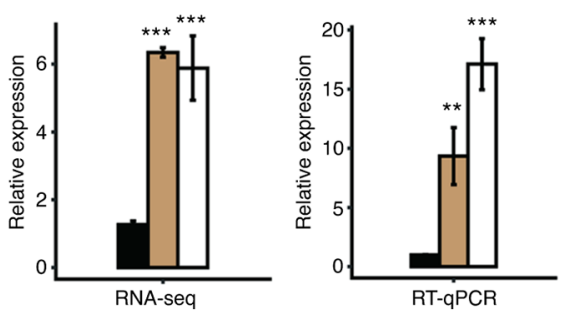

$\mathrm{IL}-1 \beta$
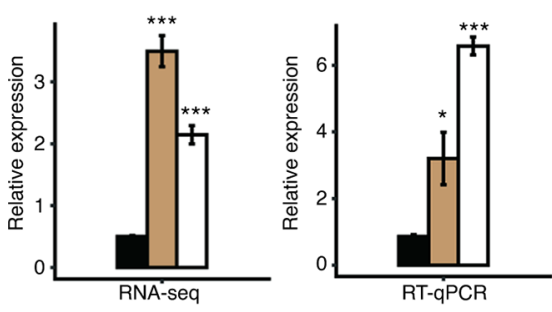

TLR2
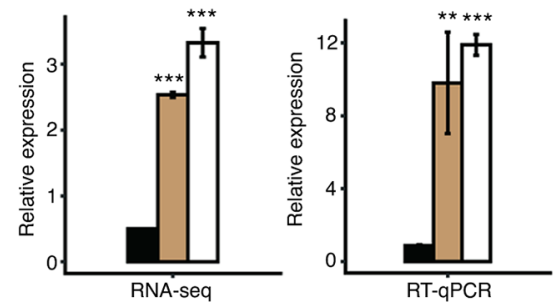

CXCL1
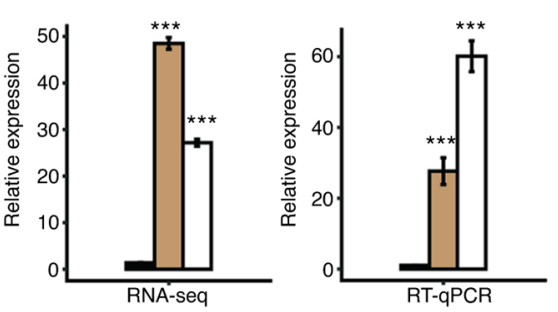

IL-34
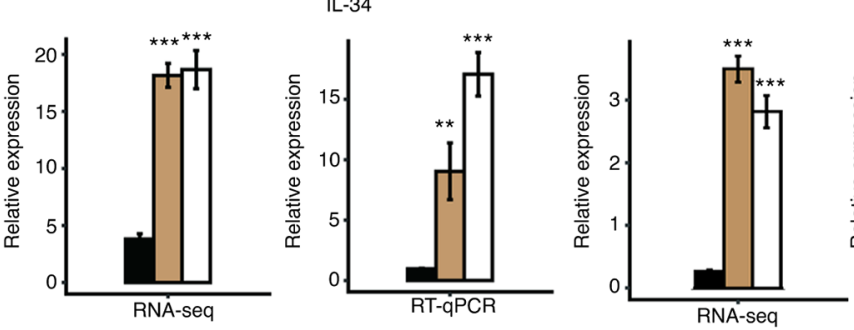

CXCL10
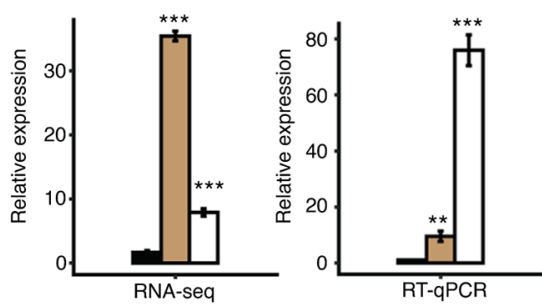

IL-6

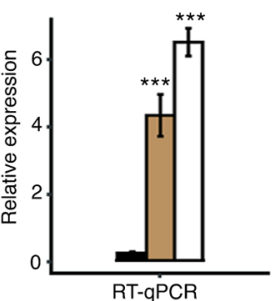

Figure 3. Validation of common differentially expressed genes related to the cisplatin and IR-induced inflammatory response pathways in acute kidney injury by reverse transcription-quantitative PCR. ${ }^{*} \mathrm{P}<0.05,{ }^{* *} \mathrm{P}<0.01$ and ${ }^{* * *} \mathrm{P}<0.001$ vs. Ctrl. CIS, cisplatin; IR, ischemia-reperfusion.

fold change of LGALS3 was in the forefront, and the expression of LGALS3 was further validated by RT-qPCR (Fig. 7B). Compared with the control group, the fold change of RBFOX1 was the highest among the downregulated RBP genes, whether in the cisplatin group or the IR group (Fig. 7C). Subsequently, this RBFOX1 trend was verified in vivo. The relative levels of RBFOX1 in mRNA and protein were significantly downregulated by cisplatin and IR (Figs. 7D and 8A). The cisplatin and IR-induced downregulation of RBFOX1 occurred in the nuclei of mouse renal tubular epithelial cells (Fig. 8B).

RBFOX1 inhibits the expression of inflammatory cytokines and the level of oxidative stress in $H K-2$ cells caused by $H / R$. Consistent with the results of animal experiments, localization analysis of the $\mathrm{HK}-2$ cells revealed that $\mathrm{H} / \mathrm{R}$ inhibited the expression of RBFOX1 in the nucleus (Fig. 8C). H/R caused the downregulation of RBFOX1 in the HK-2 cells in a time-dependent manner (Fig. 8D). The transfection efficiency of RBFOX1 plasmid in untreated and H/R-treated HK-2 cells was verified (Fig. 9A and B). Flow cytometric analysis revealed that upregulation of $\mathrm{RBFOX} 1$ reduced $\mathrm{H} / \mathrm{R}$-induced apoptosis in the HK-2 cells (Fig. 9C). In the present study, the effects of RBFOX1 on the inflammatory response and oxidative stress caused by H/R in the HK- 2 cells were analyzed. Exogenous RBFOX1 inhibited the expression of the proinflammatory cytokines TNF- $\alpha$, IL-6 and IL-1 $\beta$ induced by H/R (Fig. 9D-F). In addition, transfection of the RBFOX1 plasmid increased the resistance of the HK-2 cells to $\mathrm{H} / \mathrm{R}$-induced oxidative stress as manifested by the rebound in SOD activity and the decrease in MDA levels and ROS production (Fig. 9G-I).

RBFOX1 is capable of inhibiting $N F-\kappa B$ and activating the NRF2/HO-1 signaling pathway in H/R-induced HK-2 cells. In the present study, the effects of RBFOX 1 on $N F-\kappa B$ and the NRF2 signaling pathway in the HK-2 cells induced by $\mathrm{H} / \mathrm{R}$ were further analyzed. The results revealed that $\mathrm{H} / \mathrm{R}$ activated the expression of $\mathrm{NF}-\kappa \mathrm{B}$ and inhibited the NRF2/HO-1 signaling pathway in the HK-2 cells. Furthermore, exogenous RBFOX1 inhibited NF- $\kappa$ B but activated the NRF2/HO-1 signaling pathway (Fig. 10). These results suggested that RBFOX1 may play a protective role against inflammation and oxidative stress in H/R-induced HK-2 cells by inhibiting $\mathrm{NF}-\kappa \mathrm{B}$ and activating the NRF2/HO-1 pathway.

\section{Discussion}

AKI is a high-incidence renal disease with systemic effects and its pathogenesis is extremely complex and still not fully understood. The genome-wide bioinformatics analysis provides data basis for systematically revealing the underlying molecular mechanism of AKI and its internal associations (29). In the present study, the upregulated co-DEGs were mainly enriched in immune and inflammatory pathways, including hub genes CSF-1, CXCL1, CXCL10, IL-1 $\beta$, IL-34, IL-6 and TLR2. Importantly, the transcriptome analysis of AS and RBPs in AKI was reported, to the best of our knowledge, for the first time. Cisplatin-induced AS was enriched in 


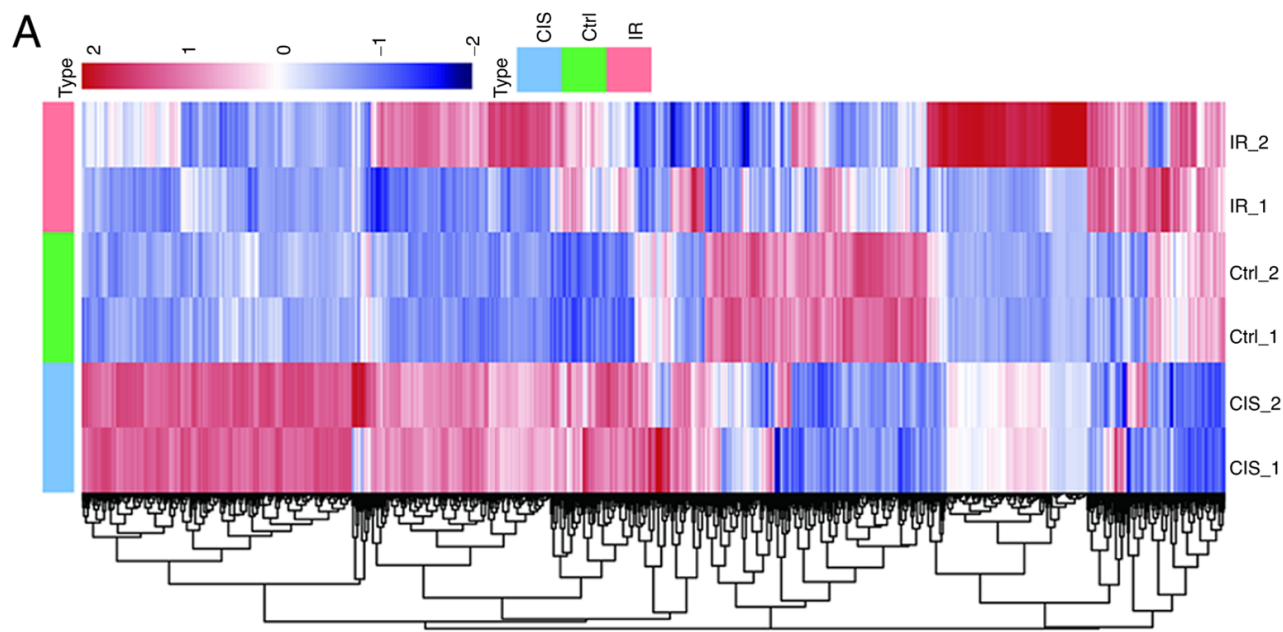

B

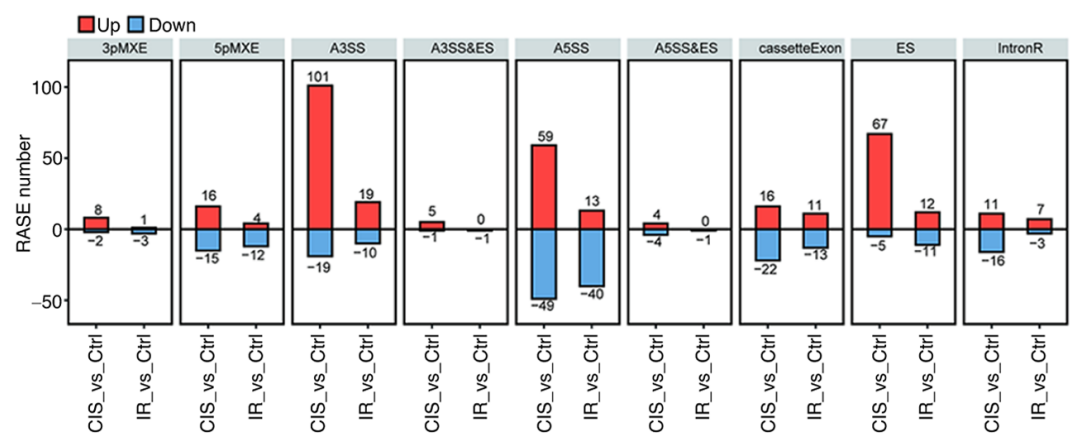

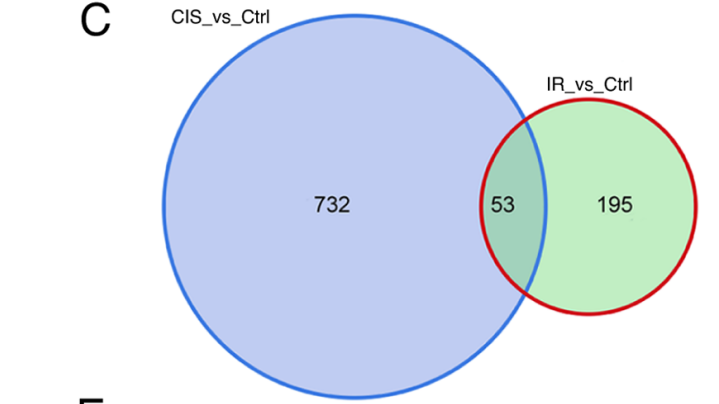

$E$

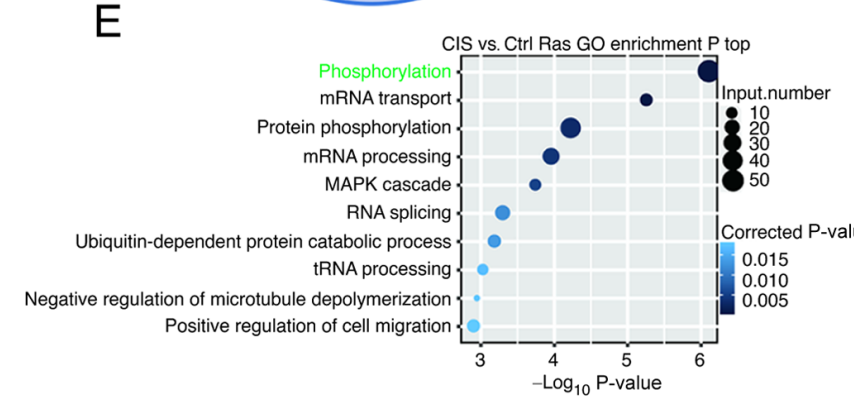

\section{G}

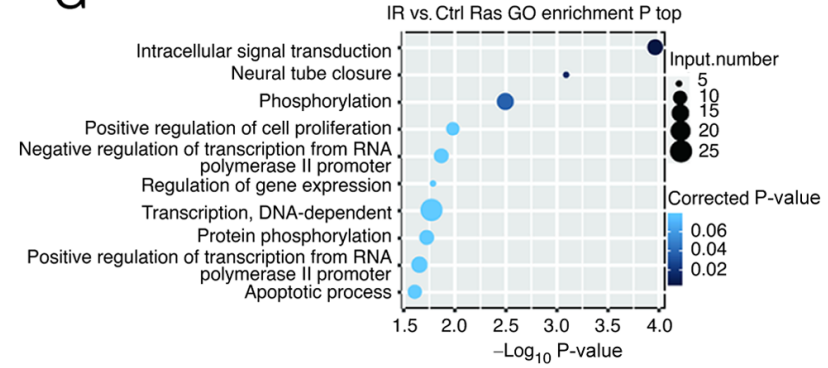

D CIS_vs_Crrl_specific_RASGs_GO_enrichment_P_top
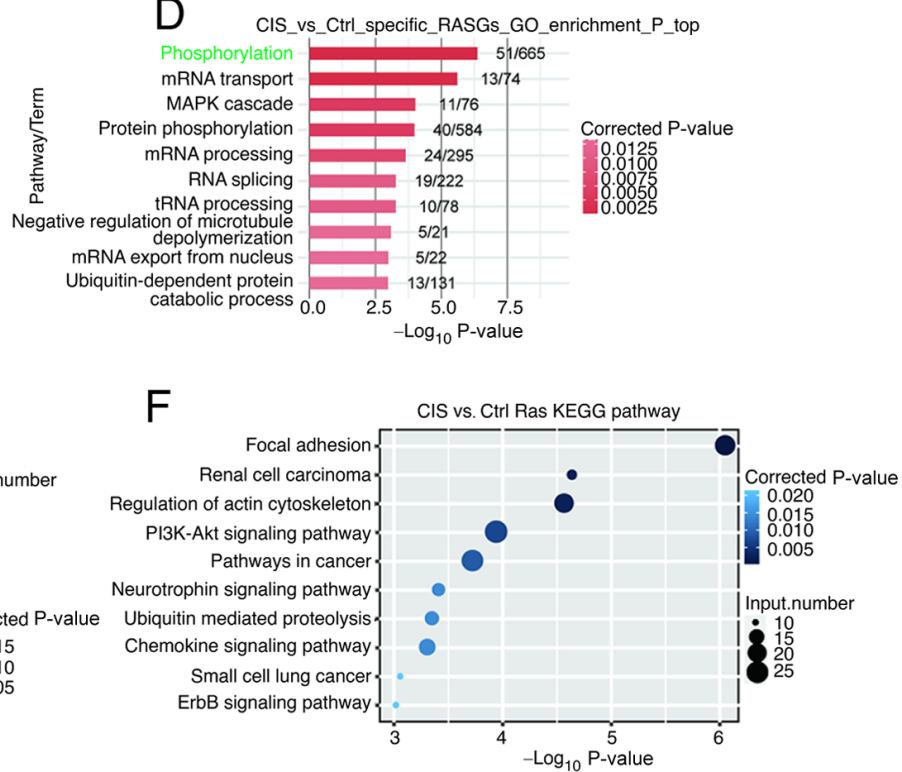

$\mathrm{H}$

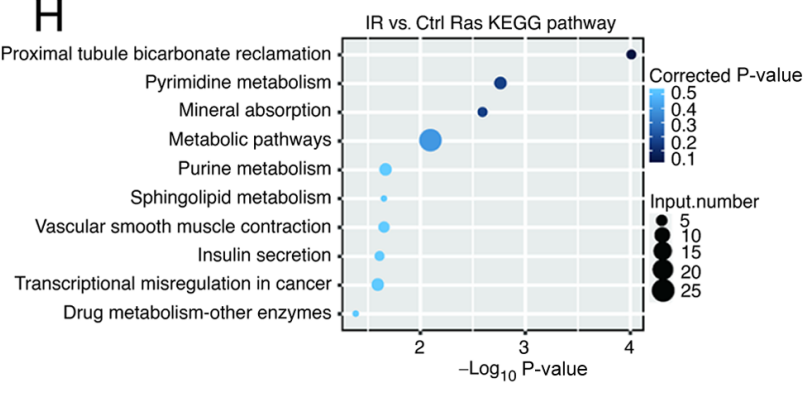

Figure 4. Transcriptome analysis of alternative splicing in AKI induced by CIS and IR. (A) Heatmap diagram revealed RASGs among CIS, IR-treated and control samples. (B) Classification of all the regulated alternative splicing events induced by CIS and IR. (C) Venn diagram showed the number of RASGs induced by CIS and IR. (D) Top 10 most enriched GO terms of CIS-specific RASGs. (E) GO analysis and (F) KEGG analysis demonstrated the top 10 most enriched terms and pathways of the RASGs in CIS-induced AKI. (G) GO analysis and (H) KEGG analysis showed the top 10 most enriched terms and pathways of the RASGs in IR-induced AKI. AKI, acute kidney injury; CIS, cisplatin; IR, ischemia-reperfusion; RASGs, regulated alternative splicing genes; GO, Gene Ontology. 

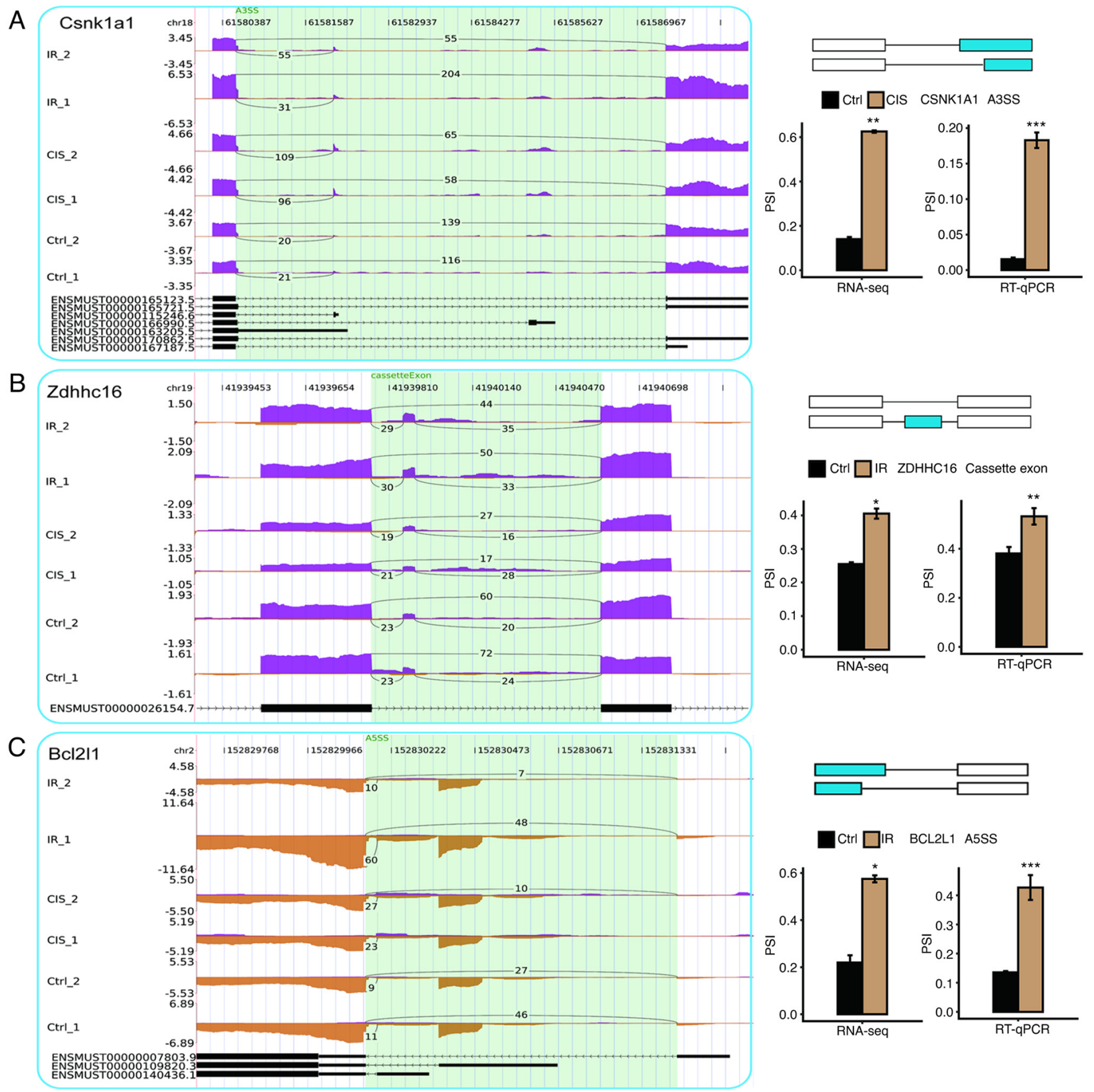

Ctrl $\square$ IR ZDHHC16 Cassette exon

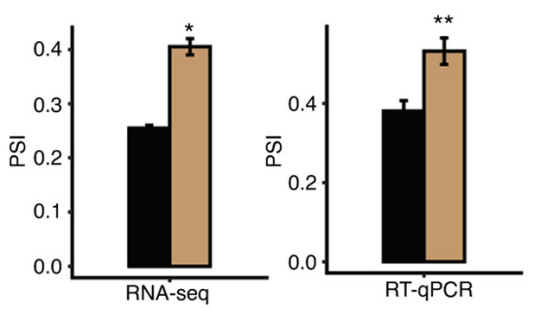

Figure 5. Examples and validation of CIS or IR-affected ASEs. (A) Alternative 3' splicing sites (A3SS), (B) cassette exon, and (C) alternative 5' splicing site (A5SS) events were shown in the IGV-sashimi plot of CSNK1A1, ZDHHC16 and BCL2L1 genes. Read distribution of each ASE was plotted in the left panel with the transcripts of each gene shown below. The schematic demonstrated the structures of ASEs. The constitutive exons are denoted by white boxes, intron sequences by a horizontal line (right panel, top), and alternative exons by blue boxes (right panel, bottom). RNA-seq quantification and reverse transcription-quantitative PCR were used to verify the ASEs. ${ }^{*} \mathrm{P}<0.05,{ }^{* *} \mathrm{P}<0.01$ and ${ }^{* * * *} \mathrm{P}<0.001$ vs. Ctrl. ASEs, alternative splicing events; CIS, cisplatin; IR, ischemia-reperfusion; PSI, percent spliced in.

the phosphorylation pathway, including DEGs CSNK1A1, PAK2, CRK, ADK and IKBKB regulated by A3SS event. IR-induced AS was enriched in the apoptosis and proliferation pathways, including RASGs FGF1 (A5SS), BCL2L1 (A5SS) and ZDHHC16 (cassette exon). Differentially expressed RBP genes may be involved in the pathogenesis of AKI. LGALS3 was the top upregulated RBP gene, whereas RBFOX1 was the top downregulated RBP gene. RBFOX1 was downregulated in the mouse kidney tissues induced by cisplatin and IR and in the HK-2 cells induced by H/R. Further experiments suggested that exogenous RBFOX1 may play a protective role against the inflammation and oxidative stress induced by $H / R$ in the HK-2 cells. This phenomenon may be related to the inhibition of $\mathrm{NF}-\kappa \mathrm{B}$ and the activation of the NRF2/HO-1 signaling pathway.

AKI is described as an inflammatory disease and inflammatory factors are involved in its damage and repair process (30). TLR 2 was originally considered to be a proinflammatory factor, but a recent study revealed that it can play a protective role in AKI by activating autophagy (31). IL-34 and CSF-1 


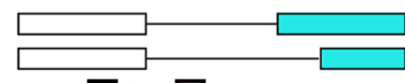

$\square$ Ctrl $\square$ CIS ADK A3SS
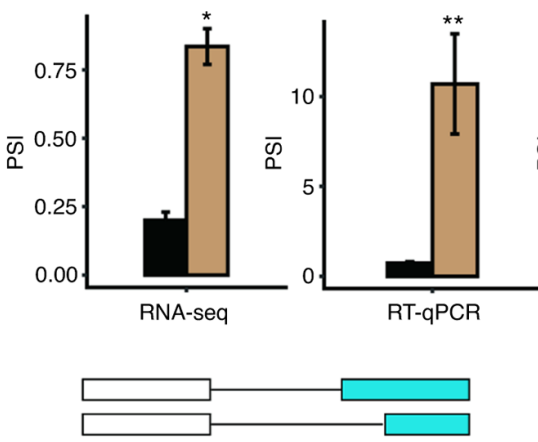

Ctrl $\square$ CIS IKBKB A3SS
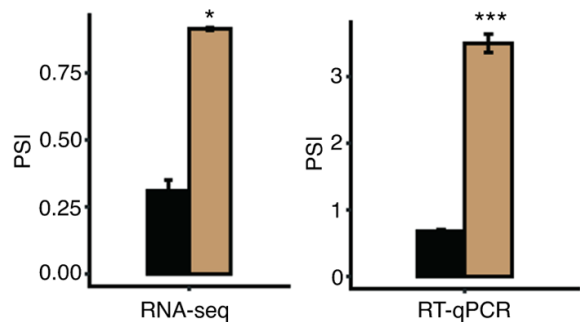

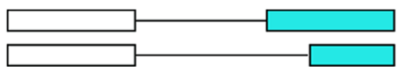

Ctrl $\square$ CIS CRK A3SS
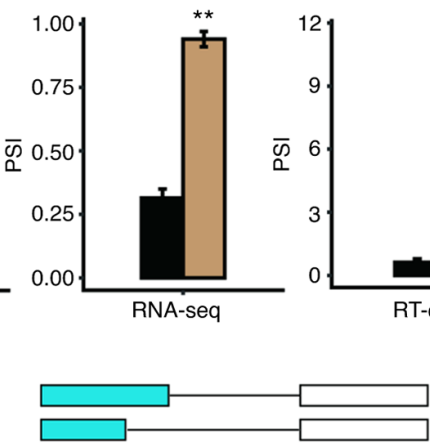

Ctrl $\square$ IR FGF1 A5SS

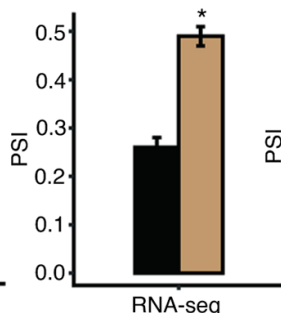

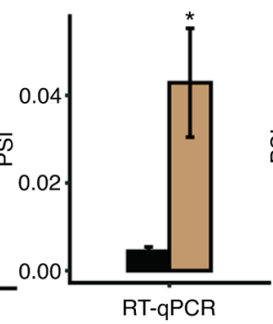

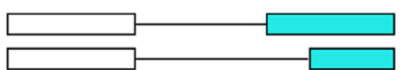

Ctrl $\square$ CIS PAK2 A3SS
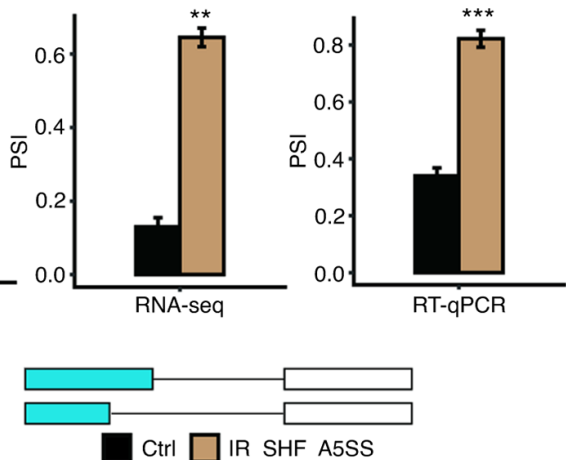

Ctrl $\square$ IR SHF A5SS
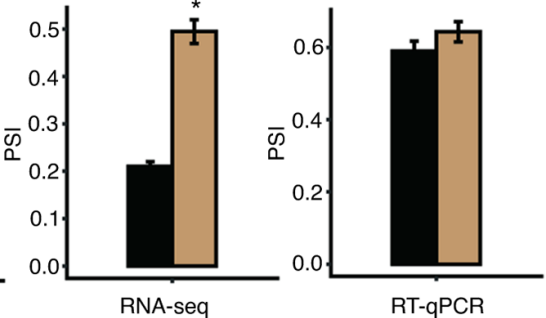

Figure 6. Validation of alternative splicing events of ADK, CRK, PAK2, IKBKB, FGF1 and SHF genes in cisplatin or IR-induced acute kidney injury by RNA-seq quantification and reverse transcription-quantitative PCR. ${ }^{*} \mathrm{P}<0.05,{ }^{* *} \mathrm{P}<0.01$ and ${ }^{* * *} \mathrm{P}<0.001$ vs. Ctrl. CIS, cisplatin; IR, ischemia-reperfusion; PSI, percent spliced in.

A
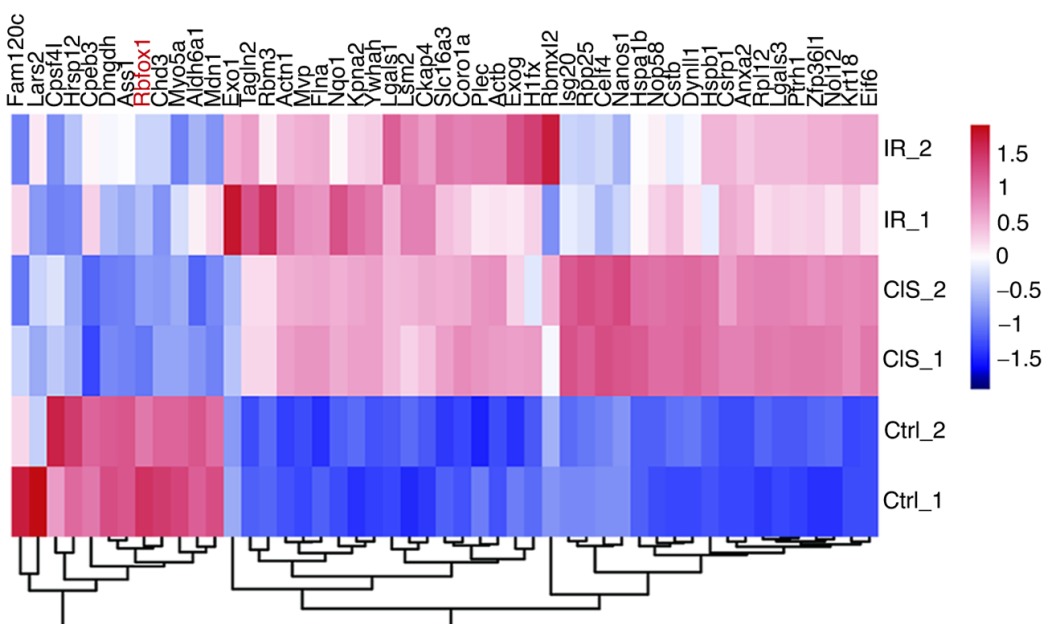

C

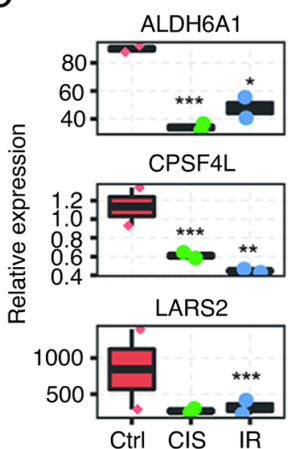

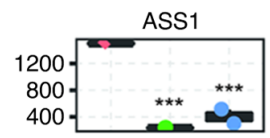
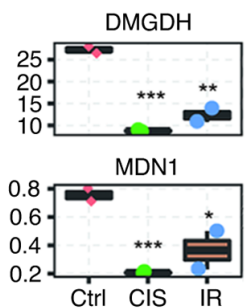
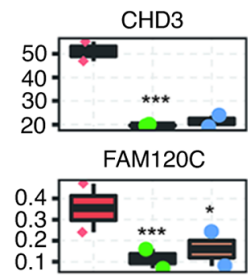

MYO5A

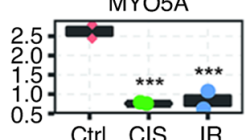

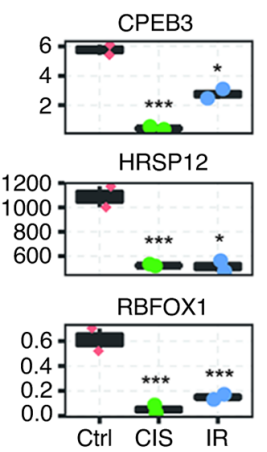

B

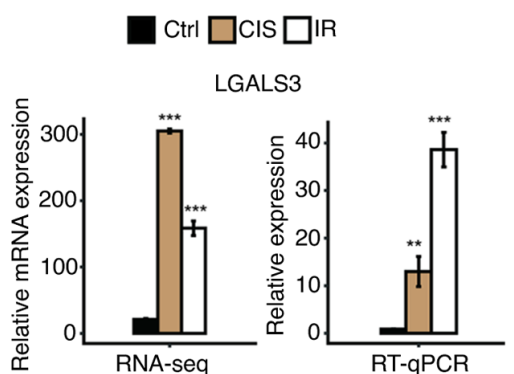

D

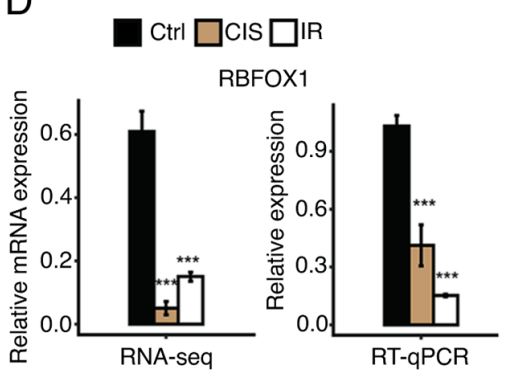

Figure 7. Expression profile and validation of RBPs in CIS and IR-induced AKI. (A) Heatmap of 49 differentially expressed RBPs in CIS and IR-induced AKI. (B) Validation of the mRNA level of LGALS3 between CIS or IR kidney samples and control. (C) Boxplots showing expression status of nine downregulated RBPs in CIS and IR-treated samples. (D) Validation of the expression of RBFOX1 between groups CIS or IR and control by reverse transcription-quantitative PCR. $\mathrm{P}<0.05$, ${ }^{* *} \mathrm{P}<0.01$ and ${ }^{* * * *} \mathrm{P}<0.001$ vs. Ctrl. RBPs, RNA binding proteins; CIS, cisplatin; IR, ischemia-reperfusion; AKI, acute kidney injury; RBFOX1, RNA binding fox-1 homolog 1 . 
A

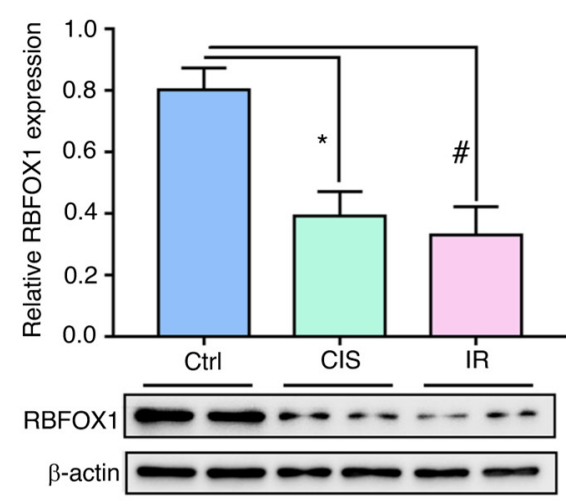

B

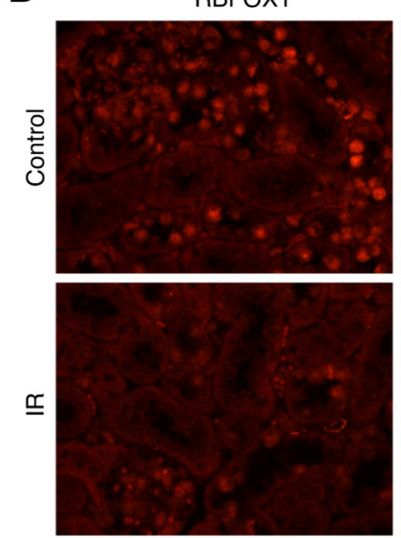

C
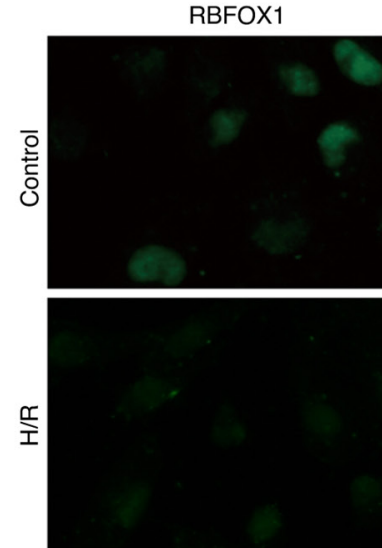

DAPI
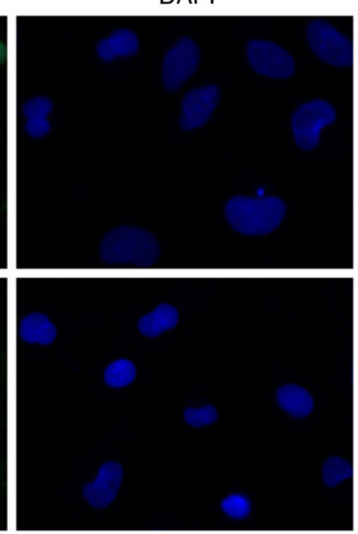

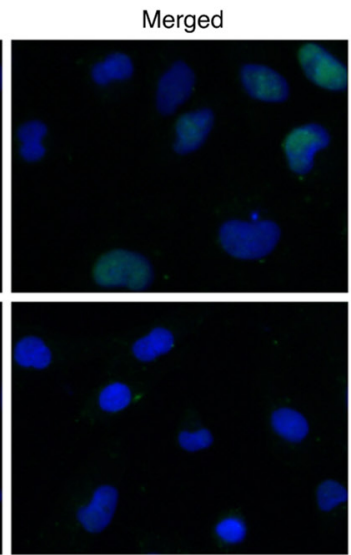

DAPI
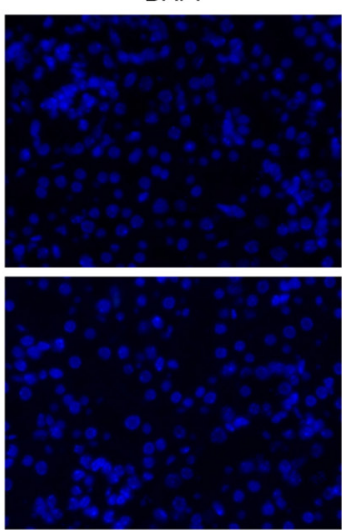

D

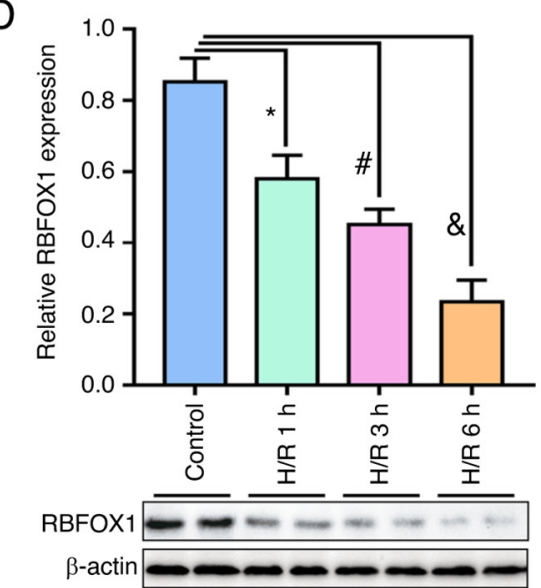

Figure 8. Changes of RBFOX1 in mouse kidney tissue and HK-2 cells. (A) Western blot analysis of RBFOX1 in mouse kidney tissue. (B and C) RBFOX1 immunofluorescence staining in mouse kidney tissue and HK-2 cells. Magnification, x200. (D) Protein expression level of RBFOX1 in HK-2 cells. * $\mathrm{P}<0.05$, ${ }^{\#} \mathrm{P}<0.05$ and ${ }^{\&} \mathrm{P}<0.05$ vs. the control. RBFOX1, RNA binding fox-1 homolog 1; CIS, cisplatin; IR, ischemia-reperfusion; H/R, hypoxia/reoxygenation.

share the same membrane receptor but have very different functions. IL-34 mediates renal tubular damage by recruiting macrophages in the acute phase of AKI and CSF-1 promotes the repair process by inducing the polarization of M2 macrophages $(32,33)$. IL-1 $\beta$ activated by inflammasomes promotes extensive inflammation in various acute and chronic kidney diseases, but the therapeutic effect of inhibiting IL-1 $\beta$ in AKI remains to be determined (34). IL-6 and CXCL1 can promote inflammatory response by inducing neutrophil infiltration in renal tissue and animal studies have shown that inhibition of IL-6 or CXCL1 has a protective effect in AKI $(35,36)$. Inflammatory factors are often used as biomarkers of AKI. Various clinical data indicated that IL-6, CXCL1 and CXCL10 in serum and urine of patients have predictive value in the early diagnosis and prognostic evaluation of AKI $(37,38)$.

The splice isoforms of CSNK1A1, ADK, CRK, PAK2 and IKBKB participate in the process of phosphorylation modification after protein translation. CSNK1A1, PAK2, and IKBKB have serine/threonine protein kinase activity and play an important role in intracellular signal transduction. Studies have demonstrated that CSNK1A1 and PAK2 phosphorylate $\beta$-catenin at Ser45 and Ser675, respectively, thereby inhibiting the WNT signaling pathway $(39,40)$. IKBKB regulates the immune response by inducing IkB-a phosphorylation at Ser32 and Ser36 through the classical activation pathway to promote $\mathrm{NF}-\kappa \mathrm{B}$ signal transduction (41). Targeted silencing of IKBKB can reduce IR-induced kidney inflammation (42). ADK regulates intracellular adenosine levels through dephosphorylation to maintain energy homeostasis. Inhibition of ADK appears to improve cisplatin-induced AKI (43). The CRK adaptor protein is the main confluence point of the phosphotyrosine kinase signaling pathway and plays a key role in maintaining the morphology and function of glomerular podocytes (44). The splice isoforms of ZDHHC16, BCL2L1 and FGF1 are closely related to cell proliferation and apoptosis. ZDHHC16 participates in post-translational modification through $\mathrm{S}$-acylation and has anti-apoptotic properties that promote cell proliferation and regulate DNA damage (45). BCL-X(L) and BCL-X(S), as the splicing isoforms of BCL2L1 (BCL-X), play anti-apoptotic and pro-apoptotic effects, respectively (46). The upregulation of $\mathrm{BCL}-\mathrm{X}(\mathrm{L})$ in the early stage of AKI indicates the existence of adaptive resistance to apoptosis (47). FGF1 mediates a precise signal cascade through autocrine/paracrine-dependent manners, participates in the regulation of cell proliferation, energy homeostasis and tissue repair and plays an anti-inflammatory effect in chronic kidney disease (48).

The strict regulation of AS requires the recruitment of RBPs. RBP can bind to pre-mRNA through a specific binding domain and plays a key role in almost all aspects of posttranscriptional regulation (49). The key RBP genes LGALS3 and RBFOX1 may play an important role in AKI. LGALS3 
A

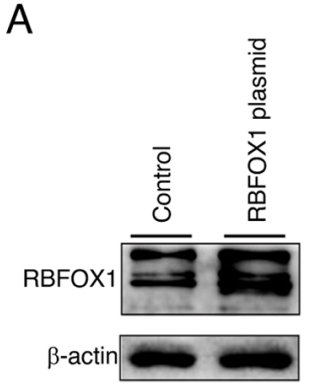

C
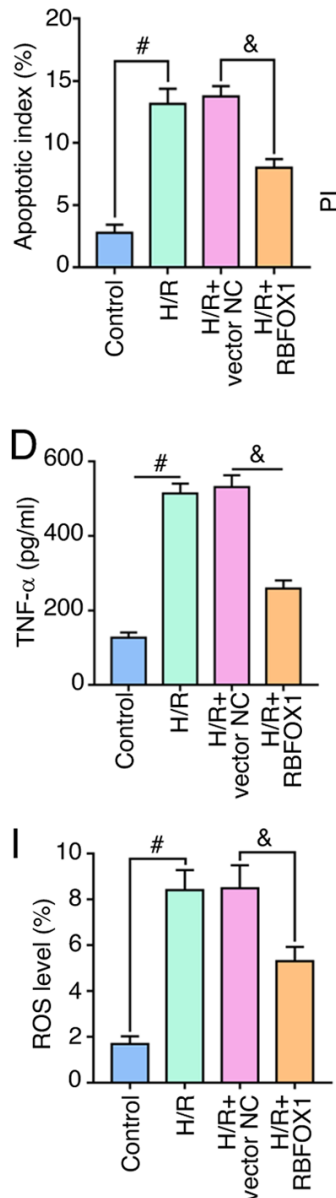

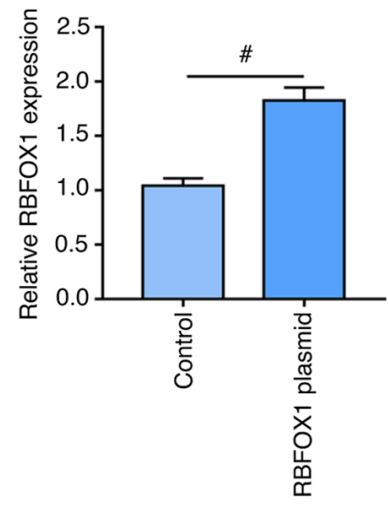

$\mathrm{B}$
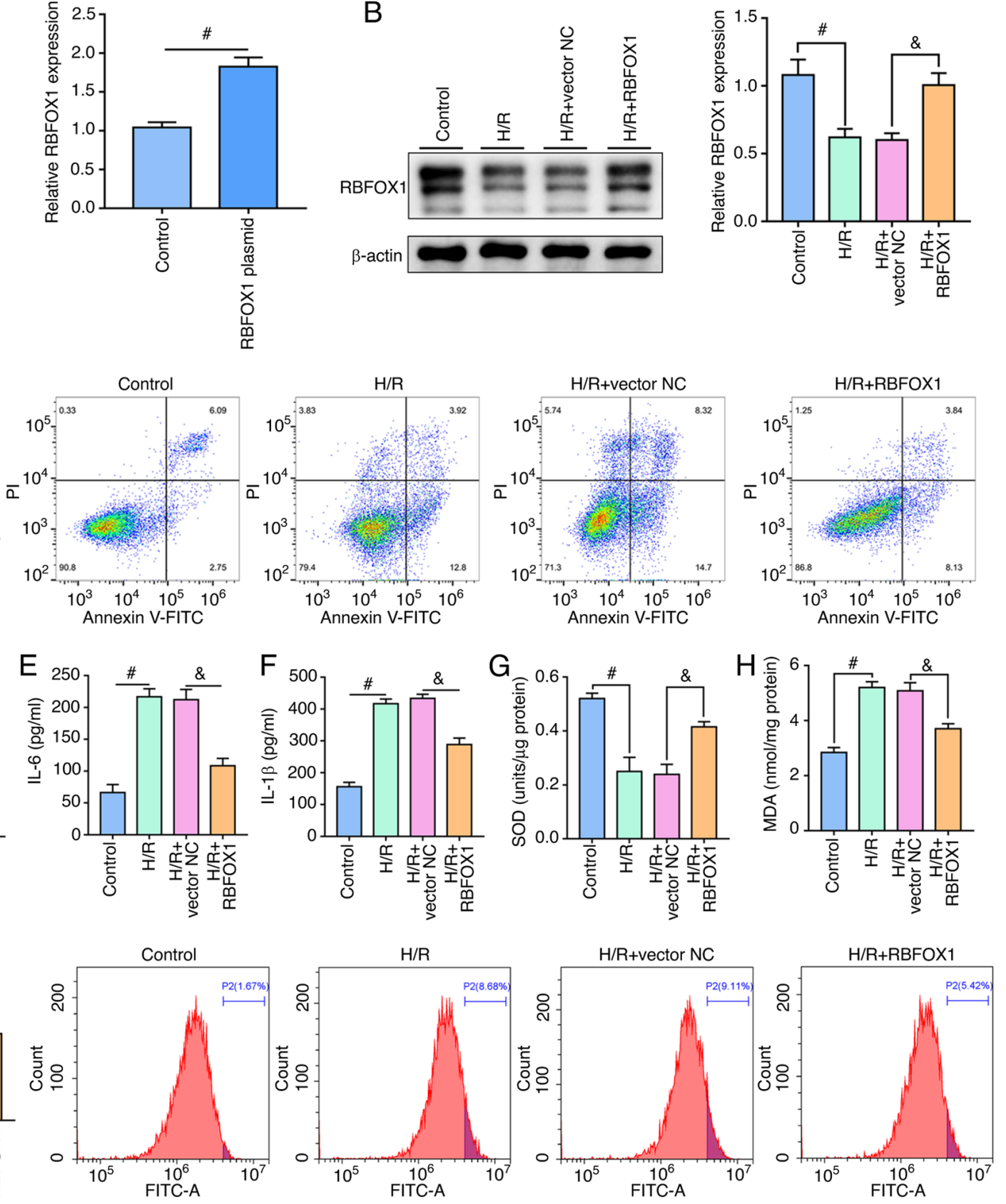

Figure 9. RBFOX1 reduces the apoptosis, acute inflammation and oxidative stress induced by H/R in HK-2 cells. (A and B) Detection of the transfection efficiency of RBFOX1 by western blotting. (C) Flow cytometric analysis of apoptosis. (D-F) Detection of TNF- $\alpha$, IL-6 and IL-1 $\beta$ for the assessment of inflammation. (G-I) SOD activity, MDA level and ROS production were detected for the assessment of oxidative stress. ${ }^{\#} \mathrm{P}<0.05$ vs. the control and ${ }^{\&} \mathrm{P}<0.05$ vs. the $\mathrm{H} / \mathrm{R}+$ vector NC. RBFOX1, RNA binding fox-1 homolog 1; H/R, hypoxia/reoxygenation; SOD, superoxide dismutase; MDA, malondialdehyde; ROS, reactive oxygen species; $\mathrm{NC}$, negative control.

is a biomarker of AKI, which can mediate inflammatory damage by regulating the migration, proliferation and activation of renal inflammatory cells (50). RBFOX1 is a highly versatile RBP composed of different splicing isomers, which can regulate transcription and affect the stability of mRNA by regulating the AS of target exons closely related to the binding motif UGCAUG (51).

Inflammation and oxidative stress play an important role in cisplatin and IR-induced AKI and studying their regulated mechanisms is of great significance to the prevention and treatment of AKI. Inhibiting $N F-\kappa B$ and activating the NRF2/HO-1 signaling pathway are important approaches by which protective genes exert anti-inflammatory and antioxidative stress effects to reduce the severity of AKI (52). As an important signal transduction factor in cells, $N F-\kappa B$ can initiate transcriptional regulation through nuclear translocation after being stimulated by external sources to promote the expression of key downstream inflammatory factors (53). The role of NF- $\kappa \mathrm{B}$ is regulated by various genes. For example, SIRT1 directly inhibits $\mathrm{NF}-\kappa \mathrm{B}$ signaling by deacetylating the p65 subunit of the NF- $\kappa \mathrm{B}$ complex (54). NRF2 is a key transcription factor of antioxidant response that induces the expression of diverse genes driven by antioxidant response elements, such as SOD and HO-1, through translocating to the nucleus 


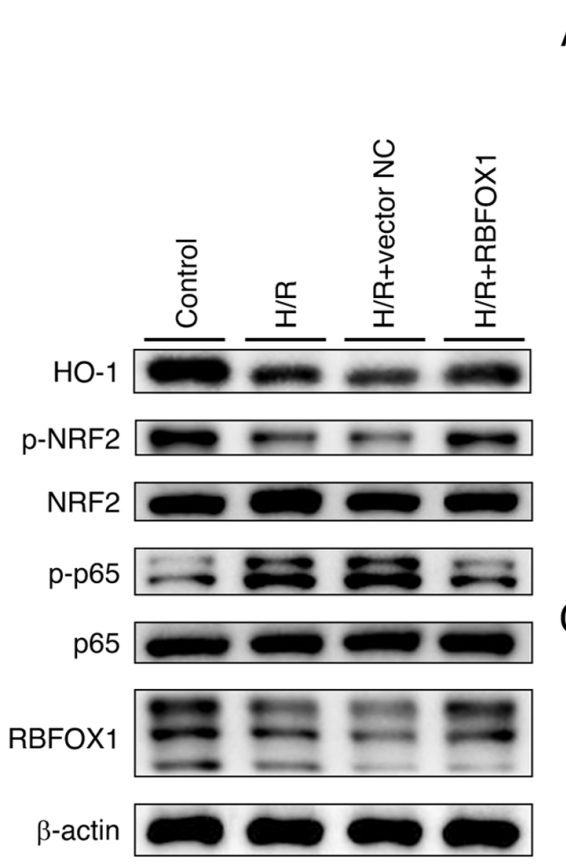

A
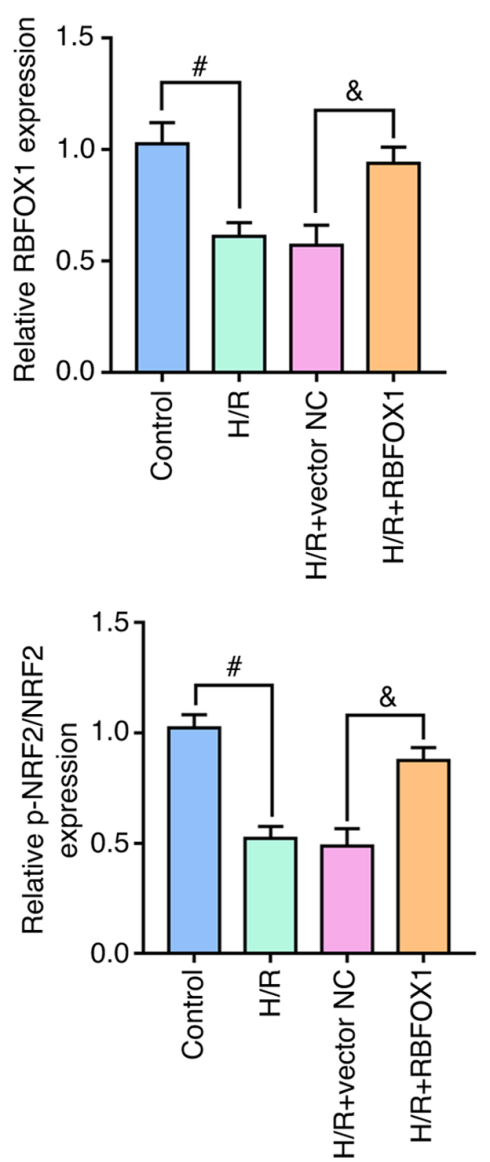

B

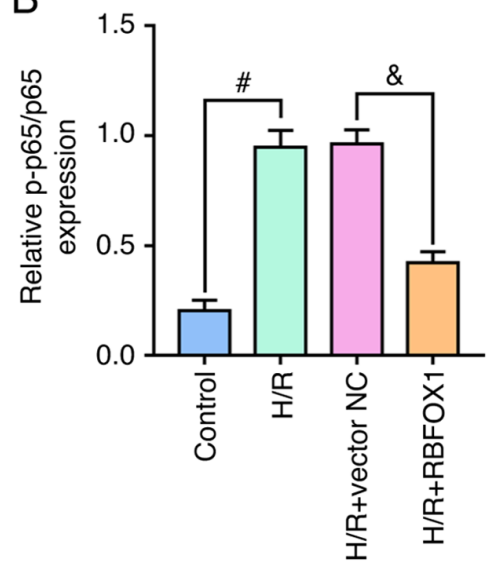

D

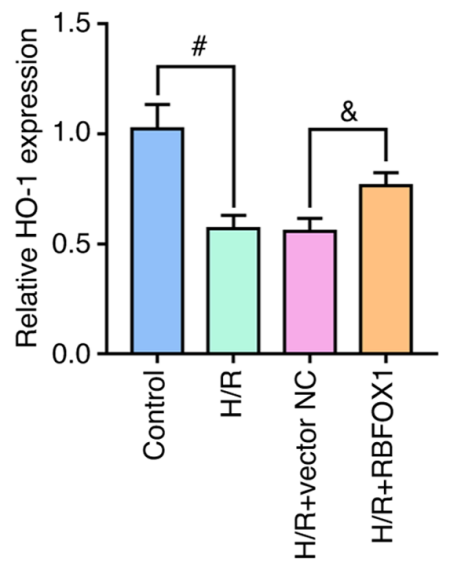

Figure 10. RBFOX1 affects the NF-kB and NRF2/HO-1 pathway in H/R-induced HK-2 cells. (A-D) Western blot analysis revealed that the transfection of RBFOX1 plasmid inhibits the upregulation of p-p65 and the downregulation of NRF2/HO-1 induced by $\mathrm{H} / \mathrm{R}$. " $\mathrm{P}<0.05$ vs. the control and ${ }^{\circledR} \mathrm{P}<0.05$ vs. the $\mathrm{H} / \mathrm{R}+$ vector NC. RBFOX1, RNA binding fox-1 homolog 1; H/R, hypoxia/reoxygenation; NC, negative control.

after deubiquitination (55). In addition to the classic KEAP1 activation, the function of NRF2 is affected by different genes. p62 can chelate KEAP1 into autophagosomes to prevent NRF2 degradation (56). It was identified that RBFOX1 is mainly expressed in the nucleus. Interestingly, both NF- $\kappa \mathrm{B}$ and NRF2 must regulate the transcription and expression of related genes through nuclear translocation. It was hypothesized that their role in the nucleus may be regulated by RBFOX1.

The present study has certain limitations. Although both cisplatin and IR were used to establish an AKI model, further increasing the amount of sequencing samples may help in revealing more possible mechanisms involved in the pathogenesis of AKI. Transcriptome sequencing and bioinformatics tools revealed the differential expression patterns of AS and RBP genes in AKI. Further molecular mechanism studies will help to link the changes in the expression of AS and RBP genes with the dysfunction caused by AKI. In the present study, the RBP gene RBFOX1, as an AS regulator, was observed to affect the expression of NF- $\mathrm{NB}$ and NRF2, but the specific mechanism of this effect remains unknown. Our future research involves the use of in vivo delivery experiments to further determine the regulatory mechanism of RBFOX1.

In the present study, the inflammatory response, AS, and RBPs in cisplatin and IR-induced AKI models were analyzed via whole transcriptome sequencing. Several hub genes, such as CSF-1, CXCL1, CXCL10, IL-1 $\beta$, IL-34, IL-6 and TLR2, were found to be mainly enriched in the immune inflammatory response and related signaling pathways. The role of AS events of the CSNK1A1, PAK2, CRK, ADK, IKBKB, ZDHHC16, BCL2L1 and FGF1 genes in the pathogenesis of AKI induced by cisplatin and IR warrants further investigation. As splicing regulators, the RBP genes, such as LGALS3 and RBFOX1, were found to be differentially expressed in the cisplatin and IR-induced AKI. RBFOX1 was downregulated in AKI and it was observed to inhibit the damage caused by inflammation and oxidative stress by affecting NF- $\kappa \mathrm{B}$ and the NRF2/HO-1 signaling pathway. These findings provided a new perspective on the mechanisms of AKI. Future studies should further elucidate the mechanistic aspects of dysregulated AS in various diseases, such as intervention in the RBP genes, as this information will provide the theoretical basis for the development of novel classes of drug splicing-modifying therapeutics.

\section{Acknowledgements}

Not applicable.

\section{Funding}

The present study was supported by grants from the National Natural Science Foundation of China (grant nos. 81870471 and 
81800617) and the Science and Technology Major Project of Hubei Province (grant nos. 2019AEA170 and 2020BCB017).

\section{Availability of data and materials}

All data generated or analyzed during this study are included in this published article. The sequencing data discussed in this publication are available under GEO Series accession number GSE142138.

\section{Authors' contributions}

FL designed the study. FL, LX and RY performed the experiments and drafted the manuscript. SH, JX, KJ and BL participated in data analysis. WY, TR, XZ and FC were involved in the discussion and interpretation of the results. $\mathrm{LX}, \mathrm{RY}, \mathrm{XZ}$ and $\mathrm{FC}$ were responsible for confirmation of the authenticity of the data. All authors have read and approved the final manuscript.

\section{Ethics approval and consent to participate}

All animal protocols were approved (approval no. WDRM20200904) by the Animal Care and Use Committee of Renmin Hospital of Wuhan University (Wuhan, China) and all animal experiments were conducted in accordance with the National Institutes of Health Guide for the Care and Use of Laboratory Animals.

\section{Patient consent for publication}

Not applicable.

\section{Competing interests}

The authors declare that they have no competing interests.

\section{References}

1. Li Q, Zhao M and Wang X: The impact of transient and persistent acute kidney injury on short-term outcomes in very elderly patients. Clin Interv Aging 12: 1013-1020, 2017.

2. Zhu H, Ren A, Zhou K, Chen Q, Zhang M and Liu J: Impact of dexmedetomidine infusion on postoperative acute kidney injury in elderly patients undergoing major joint replacement: A retrospective cohort study. Drug Des Devel Ther 14: 4695-4701, 2020

3. Brandenburger T, Salgado Somoza A, Devaux Y and Lorenzen JM Noncoding RNAs in acute kidney injury. Kidney Int 94: 870-881, 2018.

4. Hoste EAJ, Kellum JA, Selby NM, Zarbock A, Palevsky PM, Bagshaw SM, Goldstein SL, Cerdá J and Chawla LS: Global epidemiology and outcomes of acute kidney injury. Nat Rev Nephrol 14: 607-625, 2018.

5. Rabb H, Griffin MD, McKay DB, Swaminathan S, Pickkers P, Rosner MH, Kellum JA and Ronco C; Acute Dialysis Quality Initiative Consensus XIII Work Group: Inflammation in AKI: Current understanding, key questions, and knowledge gaps. J Am Soc Nephrol 27: 371-379, 2016.

6. Andrade-Oliveira V, Foresto-Neto O, Watanabe IKM, Zatz R and Camara NOS: Inflammation in renal diseases: New and old players. Front Pharmacol 10: 1192, 2019.

7. Guo Y, Ni J, Chen S, Bai M, Lin J, Ding G, Zhang Y, Sun P, Jia Z, Huang S, et al: MicroRNA-709 mediates acute tubular injury through effects on mitochondrial function. J Am Soc Nephrol 29 : 449-461, 2018.

8. Baralle FE and Giudice J: Alternative splicing as a regulator of development and tissue identity. Nat Rev Mol Cell Biol 18: 437-451, 2017.
9. Black AJ, Gamarra JR and Giudice J: More than a messenger: Alternative splicing as a therapeutic target. Biochim Biophys Acta Gene Regul Mech 1862: 194395, 2019.

10. Ule $\mathrm{J}$ and Blencowe $\mathrm{BJ}$ : Alternative splicing regulatory networks: Functions, mechanisms, and evolution. Mol Cell 76: 329-345, 2019.

11. Stevens M and Oltean S: Alternative splicing in CKD. J Am Soc Nephrol 27: 1596-1603, 2016.

12. Yee BA, Pratt GA, Graveley BR, Van Nostrand EL and Yeo GW: RBP-Maps enables robust generation of splicing regulatory maps. RNA 25: 193-204, 2019.

13. Xu Y, Ma H, Shao J, Wu J, Zhou L, Zhang Z, Wang Y, Huang Z, Ren J, Liu S, et al: A role for tubular necroptosis in cisplatin-induced AKI. J Am Soc Nephrol 26: 2647-2658, 2015.

14. Xiao L, Zhou D, Tan RJ, Fu H, Zhou L, Hou FF and Liu Y: Sustained activation of Wnt/beta-catenin signaling drives AKI to CKD progression. J Am Soc Nephrol 27: 1727-1740, 2016.

15. Yu X, Meng X, Xu M, Zhang X, Zhang Y, Ding G, Huang S, Zhang A and Jia Z: Celastrol ameliorates cisplatin nephrotoxicity by inhibiting NF- $\mathrm{KB}$ and improving mitochondrial function. EBioMedicine 36: 266-280, 2018.

16. Zhou X, Jiang K, Luo H, Wu C, Yu W and Cheng F: Novel lncRNA XLOC_032768 alleviates cisplatin-induced apoptosis and inflammatory response of renal tubular epithelial cells through TNF- $\alpha$. Int Immunopharmacol 83: 106472, 2020.

17. Kim D, Pertea G, Trapnell C, Pimentel H, Kelley R and Salzberg SL: TopHat2: Accurate alignment of transcriptomes in the presence of insertions, deletions and gene fusions. Genome Biol 14: R36, 2013.

18. Trapnell C, Williams BA, Pertea G, Mortazavi A, Kwan G, van Baren MJ, Salzberg SL, Wold BJ and Pachter L: Transcript assembly and quantification by RNA-Seq reveals unannotated transcripts and isoform switching during cell differentiation. Nat Biotechnol 28: 511-515, 2010.

19. Robinson MD, McCarthy DJ and Smyth GK: edgeR: A Bioconductor package for differential expression analysis of digital gene expression data. Bioinformatics 26: 139-140, 2010.

20. Song Q, Yi F, Zhang Y, Li DK, Wei Y, Yu H and Zhang Y: CRKL regulates alternative splicing of cancer-related genes in cervical cancer samples and HeLa cell. BMC Cancer 19: 499, 2019.

21. Livak KJ and Schmittgen TD: Analysis of relative gene expression data using real-time quantitative PCR and the 2(-Delta Delta C(T)) method. Methods 25: 402-408, 2001.

22. Tu Y, Wu X, Yu F, Dang J, Wang J, Wei Y, Cai Z, Zhou Z, Liao W, Li L and Zhang Y: Tristetraprolin specifically regulates the expression and alternative splicing of immune response genes in HeLa cells. BMC Immunol 20: 13, 2019.

23. Ashburner M, Ball CA, Blake JA, Botstein D, Butler $\mathrm{H}$, Cherry JM, Davis AP, Dolinski K, Dwight SS, Eppig JT, et al: Gene ontology: Tool for the unification of biology. The gene ontology consortium. Nat Genet 25: 25-29, 2000.

24. Kanehisa M, Furumichi M, Sato Y, Ishiguro-Watanabe $M$ and Tanabe M: KEGG: Integrating viruses and cellular organisms. Nucleic Acids Res 49: D545-D551, 2021.

25. Xie C, Mao X, Huang J, Ding Y, Wu J, Dong S, Kong L, Gao G, Li CY and Wei L: KOBAS 2.0: A web server for annotation and identification of enriched pathways and diseases. Nucleic Acids Res 39: W316-W322, 2011.

26. Shannon P, Markiel A, Ozier O, Baliga NS, Wang JT, Ramage D, Amin N, Schwikowski B and Ideker T: Cytoscape: A software environment for integrated models of biomolecular interaction networks. Genome Res 13: 2498-2504, 2003.

27. Liu C, Chen K, Wang H, Zhang Y, Duan X, Xue Y, He H, Huang Y, Chen Z, Ren H, et al: Gastrin attenuates renal ischemia/reperfusion injury by a PI3K/Akt/bad-mediated anti-apoptosis signaling. Front Pharmacol 11: 540479, 2020.

28. Zhang Y, Zhang JJ, Liu XH and Wang L: CBX7 suppression prevents ischemia-reperfusion injury-induced endoplasmic reticulum stress through the Nrf-2/HO-1 pathway. Am J Physiol Renal Physiol 318: F1531-F1538, 2020.

29. Marx D, Metzger J, Pejchinovski M, Gil RB, Frantzi M, Latosinska A, Belczacka I, Heinzmann SS, Husi H, Zoidakis J, et al: Proteomics and metabolomics for AKI diagnosis. Semin Nephrol 38: 63-87, 2018.

30. Sabapathy V, Venkatadri R, Dogan M and Sharma R: The Yin and Yang of alarmins in regulation of acute kidney injury. Front Med (Lausanne) 7: 441, 2020.

31. Andrade-Silva M, Cenedeze MA, Perandini LA, Felizardo RJF, Watanabe IKM, Agudelo JSH, Castoldi A, Gonçalves GM, Origassa CST, Semedo P, et al: TLR2 and TLR4 play opposite role in autophagy associated with cisplatin-induced acute kidney injury. Clin Sci (Lond) 132: 1725-1739, 2018. 
32. Baek JH, Zeng R, Weinmann-Menke J, Valerius MT, Wada Y, Ajay AK, Colonna M and Kelley VR: IL-34 mediates acute kidney injury and worsens subsequent chronic kidney disease. J Clin Invest 125: 3198-3214, 2015.

33. Zhang MZ, Yao B, Yang S, Jiang L, Wang S, Fan X, Yin $H$, Wong K, Miyazawa T, Chen J, et al: CSF-1 signaling mediates recovery from acute kidney injury. J Clin Invest 122: 4519-4532, 2012.

34. Anders HJ: Of inflammasomes and alarmins: IL-1 $\beta$ and IL-1 $\alpha$ in kidney disease. J Am Soc Nephrol 27: 2564-2575, 2016.

35. Nechemia-Arbely Y, Barkan D, Pizov G, Shriki A, Rose-John S, Galun E and Axelrod JH: IL-6/IL-6R axis plays a critical role in acute kidney injury. J Am Soc Nephrol 19: 1106-1115, 2008.

36. Liu P, Li X, Lv W and Xu Z: Inhibition of CXCL1-CXCR2 axis ameliorates cisplatin-induced acute kidney injury by mediating inflammatory response. Biomed Pharmacother 122: 109693 , 2020.

37. Zhang WR, Garg AX, Coca SG, Devereaux PJ, Eikelboom J, Kavsak P, McArthur E, Thiessen-Philbrook H, Shortt C, Shlipak M, et al: Plasma IL-6 and IL-10 concentrations predict AKI and long-term mortality in adults after cardiac surgery. J Am Soc Nephrol 26: 3123-3132, 2015.

38. Erez DL, Denburg MR, Afolayan S, Jodele S, Wallace G, Davies SM, Seif AE, Bunin N, Laskin BL and Sullivan KE: Acute kidney injury in children after hematopoietic cell transplantation is associated with elevated urine CXCL10 and CXCL9. Biol Blood Marrow Transplant 26: 1266-1272, 2020.

39. Jiang S, Zhang M, Sun J and Yang X: Casein kinase $1 \alpha$ : Biological mechanisms and theranostic potential. Cell Commun Signal 16: 23, 2018.

40. Peng X, Lai KS, She P, Kang J, Wang T, Li G, Zhou Y, Sun J, Jin D, Xu X, et al: Induction of Wnt signaling antagonists and p21-activated kinase enhances cardiomyocyte proliferation during zebrafish heart regeneration. J Mol Cell Biol 13: 41-58, 2021.

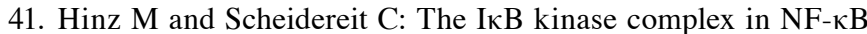
regulation and beyond. EMBO Rep 15: 46-61, 2014.

42. Wan X, Fan L, Hu B, Yang J, Li X, Chen X and Cao C: Small interfering RNA targeting IKK $\beta$ prevents renal ischemia-reperfusion injury in rats. Am J Physiol Renal Physiol 300: F857-F863, 2011.

43. Cao W, Yuan Y, Liu X, An X, Huang Z, Wu L, Zhang B, Zhang A and Xing C: Adenosine kinase inhibition protects against cisplatin-induced nephrotoxicity. Am J Physiol Renal Physiol 317: F107-F115, 2019.

44. Du J, Meng L, Pang L, Jin B, Duan N, Huang C, Huang H and $\mathrm{Li} \mathrm{H}$ : Crk1/2 and CrkL play critical roles in maintaining podocyte morphology and function. Exp Cell Res 394: 112135, 2020.
45. Cao N, Li JK, Rao YQ, Liu H, Wu J, Li B, Zhao P, Zeng L and Li J: A potential role for protein palmitoylation and zDHHC16 in DNA damage response. BMC Mol Biol 17: 12, 2016.

46. Stevens M and Oltean S: Modulation of the apoptosis gene Bcl-x function through alternative splicing. Front Genet 10: 804, 2019

47. Valdes F, Pasaro E, Diaz I, Centeno A, López E, García-Doval S, González-Roces S, Alba A and Laffon B: Segmental heterogeneity in $\mathrm{Bcl}-2, \mathrm{Bcl}-\mathrm{xL}$ and $\mathrm{Bax}$ expression in rat tubular epithelium after ischemia-reperfusion. Nephrology (Carlton) 13: 294-301, 2008.

48. Liang G, Song L, Chen Z, Qian Y, Xie J, Zhao L, Lin Q, Zhu G, Tan Y, Li X, et al: Fibroblast growth factor 1 ameliorates diabetic nephropathy by an anti-inflammatory mechanism. Kidney Int 93: 95-109, 2018

49. Corley M, Burns MC and Yeo GW: How RNA-binding proteins interact with RNA: Molecules and mechanisms. Mol Cell 78: 9-29, 2020.

50. Sun H, Jiang H, Eliaz A, Kellum JA, Peng Z and Eliaz I: Galectin-3 in septic acute kidney injury: A translational study. Crit Care 25: 109, 2021.

51. Conboy JG: Developmental regulation of RNA processing by Rbfox proteins. Wiley Interdiscip Rev RNA 8: 10.1002/wrna.1398, 2017.

52. Xiang H, Xue W, Li Y, Zheng J, Ding C, Dou M and Wu X: Knockdown of ANGPTL2 protects renal tubular epithelial cells against hypoxia/reoxygenation-induced injury via suppressing TLR4/NF- $\kappa \mathrm{B}$ signaling pathway and activating Nrf2/HOsignaling pathway. Cell Transplant 29: 963689720946663, 2020

53. Song N, Thaiss F and Guo L: NFkB and kidney injury. Front Immunol 10: 815, 2019.

54. Yeung F, Hoberg JE, Ramsey CS, Keller MD, Jones DR, Frye RA and Mayo MW: Modulation of NF-kappaB-dependent transcription and cell survival by the SIRT1 deacetylase. EMBO J 23: 2369-2380, 2004.

55. Wei W, Ma N, Fan X, Yu Q and Ci X: The role of Nrf2 in acute kidney injury: Novel molecular mechanisms and therapeutic approaches. Free Radic Biol Med 158: 1-12, 2020.

56. Komatsu M, Kurokawa H, Waguri S, Taguchi K, Kobayashi A Ichimura Y, Sou YS, Ueno I, Sakamoto A, Tong KI, et al: The selective autophagy substrate p62 activates the stress responsive transcription factor Nrf2 through inactivation of Keap1. Nat Cell Biol 12: 213-223, 2010.

This work is licensed under a Creative Commons Attribution-NonCommercial-NoDerivatives 4.0 International (CC BY-NC-ND 4.0) License. 